\title{
Two-step weighting method for constructing fourth-order hybrid central WENO scheme
}

\author{
Shengping Liu ${ }^{\mathrm{a}}$, Yiqing Shen ${ }^{\mathrm{b}, \mathrm{c}}$, Jun Peng ${ }^{\mathrm{b}, \mathrm{c}}$, Jun Zhang ${ }^{\mathrm{a}, *}$ \\ a School of Aeronautic Science and Engineering, Beihang University, Beijing 100191, China \\ ${ }^{\mathrm{b}}$ State Key Laboratory of High Temperature Gas Dynamics, Institute of Mechanics, Chinese Academy of Sciences, Beijing 100190, China \\ ' School of Engineering Science, University of Chinese Academy of Sciences, Beijing 100049, China
}

\section{A R T I C L E I N F O}

\section{Article history:}

Received 24 January 2020

Revised 5 May 2020

Accepted 25 May 2020

Available online 26 May 2020

\section{Keywords:}

Hybrid central WENO scheme

Discontinuity-detecting method

Two-step weighting method

\begin{abstract}
A B S T R A C T
The hybridization of the fourth-order central scheme and a third-order WENO (weighted essentially nonoscillatory) scheme aims to obtain fourth-order accuracy in smooth regions and keep ENO property near shock waves. However, according to our numerical tests, such existing hybrid schemes may generate spurious waves near shocks. To overcome this problem, in this paper, a new two-step method is proposed for constructing a fourth-order hybrid central WENO scheme. The first step is using the traditional weighting method to get the third-order WENO scheme. The second step is to get a hybrid scheme by weighting the third-order upwind scheme and the fourth-order central scheme. Then, the final hybrid WENO scheme is obtained by combining the two weighted schemes through a discontinuity-detecting method for a four-point stencil. Various one- and two-dimensional numerical tests are presented to show that the new scheme can avoid the generation of spurious waves near shock waves, keep the ENO property, and get almost fourth-order accuracy in smooth regions including critical points.
\end{abstract}

(c) 2020 Elsevier Ltd. All rights reserved.

\section{Introduction}

Due to the high-order accuracy and the essentially nonoscillatory (ENO) property, the WENO schemes have been widely applied in many areas [1]. The WENO scheme was firstly proposed by Liu et al.[2], and then greatly simplified and improved by Jiang and Shu[3](WENO-JS). Within the general framework of the smoothness indicators and the nonlinear weights design of Jiang and $\mathrm{Shu}[3]$, many efforts were made to further improve the WENO scheme. Balsara and Shu[4] extended the WENO scheme up to 11th order accuracy, Gerolymos et al.[5] further developed very-highorder WENO schemes. Henrick et al.[6] derived the necessary and sufficient conditions for the fifth-order convergence of a fifth-order WENO scheme and pointed out that the weights of WENO-JS cannot meet the conditions at critical points, hence they proposed a mapping function to improve its accuracy. Later, Borges et al.[7] introduced a fifth-order global smoothness indicator and designed the WENO-Z scheme, which can improve the accuracy in smooth regions and decrease the numerical dissipation near shock regions with less computational cost than the mapping function[6]. And,

\footnotetext{
* Corresponding author.

E-mail address: jun.zhang@buaa.edu.cn (J. Zhang).
}

the works in Ha et al. [8], Fan et al. [9], Kim et al. [10], Acker et al. [11], Liu et al. [12] showed that developing higher-order global smoothness indicators and modifying the weighting function can further improve the fifth-order WENO-Z scheme. Castro et al.[13] also developed higher-order WENO-Z schemes. Shen and Zha[14] noticed that the accuracy of the above mentioned WENO schemes would decrease at transitional points, which connect a smooth region and a discontinuity, and proposed a multi-step weighting method to fix this problem. Lately, Ma et al.[15], Zeng et al.[16] simplified the multi-step weighting method and reduced its computational cost. Hejranfar et al.[17] developed high order WENO formulation of the lattice Boltzmann method in generalized curvilinear coordinates. This method includes a linear equation which makes its programming much easier than $\mathrm{N}-\mathrm{S}$ based solvers $[18,19]$. More works about the accuracy, numerical dissipation, efficiency and implementation of the WENO schemes can be found in Shen and Zha [20], Xu and Shu [21], Huang and Chen [22], Johnsen and Colonius [23], He et al. [24], Zhao et al. [25], Peng et al. [26].

In general, for a smooth solution, a central scheme is more ideal than an upstream (or upwind) scheme. Hence, there are many efforts to conjugate shock-capturing schemes, to be applied around discontinuities, with high-order central finite difference methods 
for the smooth parts of the solution, such as, the hybrid central WENO scheme developed in Kim and Kwon [27], Costa and Don [28], Hu et al. [29], Huang and Chen [30], Yamaleev and Carpenter [31], Hu [32], Martin et al. [33]. In early works, Adams and Shariff[34], Ren et al.[35] noticed that, for those hybrid schemes, the abrupt switch from a shock-capturing scheme (e.g., the ENO or WENO schemes) to a compact scheme at the interface between smooth regions and discontinuities may result in spurious waves. Ren et al.[35] suggested using a weight function to avoid the abrupt transition from one sub-scheme to another, the resulting weighted hybrid scheme with the weight function is essentially oscillation free near discontinuities. Kim and Kwon[27] developed the weighted hybrid central WENO scheme with an alternative weight function. By taking account of the contribution of the downwind stencil, Martin et al.[33] developed the WENOSYMOO and WENO-SYMBO schemes. Hu et al.[29] constructed an adaptive central-upwind WENO scheme. Costa et al.[28] pointed out that, due to the non-dissipative nature of the central finite difference scheme, the inherent dispersion error, although small, generates undesirable oscillations polluting the solution of central WENO schemes in time.

Compared with higher-order WENO schemes, the third-order one has several advantages. For example, it is more robust for shock problems, it uses less grid points, and hence, it reduces the difficulty of the boundary treatment and can be easily generalized to unstructured meshes, and it provides a suitable compromise of the computation cost and the accuracy in some cases [36,37]. Recently, many works were proposed to improve its performance [36-45]. However, the analysis and numerical results [12,37] show that these third-order WENO schemes always fail to obtain expected order near critical points. And Liu et al.[37] pointed out that one important reason for the accuracy losing of these third-order WENO schemes is that their local smoothness indicators cannot correctly treat the smooth three-point stencil containing a nonnodal critical point, here, non-nodal means the critical point is not a grid point. To improve the accuracy of a third-order WENO scheme, a third-order hybrid WENO scheme was proposed in Liu and Shen [37] by combining the third-order WENO scheme and a third-order upwind scheme via a discontinuity-detecting method for a four-point stencil. It is worthy to point out that, even a fourpoint stencil is used in Liu and Shen [37], the fourth-order central scheme on this stencil cannot be directly applied to replace the third-order upwind scheme, otherwise, some spurious numerical phenomena may be generated, for example, oscillation, apparent asymmetry, even less third-order accuracy in smooth regions. This is why only the third-order upwind scheme is used in Liu and Shen [37]. Wu et al.[39] constructed a hybrid scheme of a thirdorder WENO-N3 scheme and a fourth-order central scheme with a modified weight function of Ren et al.[35]. As our numerical results show, this hybrid central WENO scheme [39] generates spurious structures near shocks.

In order to take advantage of the fourth-order central scheme and suppress the generation of spurious phenomena near shocks, in this paper, we propose a new two-step method for constructing a fourth-order hybrid central WENO scheme, in which the thirdorder WENO-MN3[36] or a weighted scheme of the third-order upwind and the fourth-order central schemes is chosen according to the discontinuity-detecting method for a four-point stencil [37]. For simplicity, the new scheme is called as HWENO-U3C4 in this paper.

This paper is organized as follows: several third-order WENO schemes [3,36,39] and the hybrid WENO schemes [37,39] are briefly introduced in Section 2. The new HWENO-U3C4 scheme is given in Section 3. In Section 4, various numerical examples are presented to demonstrate the good performance of HWENO-U3C4. Concluding remarks are given in Section 5.

\section{The third-order WENO and hybrid WENO schemes}

In this section, we briefly review the third-order WENO-JS3 [3], WENO-N3 [39], WENO-MN3 [36] and two hybrid WENO schemes(HWENO-N3 [39] and S-HWMN3 [37]) by using the scalar conservative law equation [3],

$\frac{\partial u}{\partial t}+\frac{\partial f}{\partial x}=0$

where $u(x, t)$ is a conserved quantity, $f(u(x, t))$ describes its flux, and $x$ and $t$ denote space and time, respectively. By defining the points $x_{i}=i \Delta x,(i=0, \ldots, N)$, where $\Delta x$ is the uniform grid spacing, the Eq. (1) can be approximated by a conservative finite difference formula,

$$
\frac{d u_{i}}{d t}=-\frac{\hat{f}_{i+1 / 2}-\hat{f}_{i-1 / 2}}{\Delta x},
$$

where $\hat{f}_{i \pm 1 / 2}$ is the numerical flux. Generally, the flux can be split into positive part and negative part,

$\hat{f}_{i+1 / 2}=\hat{f}_{i+1 / 2}^{+}+\hat{f}_{i+1 / 2}^{-}$.

Here, only the positive part $\hat{f}_{i+1 / 2}^{+}$is described, and the superscript "+" is dropped for simplicity. The negative part $\hat{f}_{i+1 / 2}^{-}$is symmetric with respect to $x_{i+1 / 2}$ and will not be shown.

\subsection{The WENO-JS and WENO-N3 schemes}

The flux $\hat{f}_{i+1 / 2}$ of a third-order WENO scheme can be written as,

$\hat{f}_{i+1 / 2}=\omega_{0} q_{0}+\omega_{1} q_{1}$,

where, $q_{k}$ is the second-order flux on the sub-stencil $S_{k}=(i-1+$ $k, i+k)$ and is given by,

$\left\{\begin{array}{l}q_{0}=-\frac{1}{2} f_{i-1}+\frac{3}{2} f_{i}, \\ q_{1}=\frac{1}{2} f_{i}+\frac{1}{2} f_{i+1},\end{array}\right.$

The weight $\omega_{k}$ of the WENO-JS3 [3] is constructed as,

$\omega_{k}=\frac{\alpha_{k}}{\alpha_{0}+\alpha_{1}}, \alpha_{k}=\frac{c_{k}}{\left(I S_{k}+\epsilon\right)^{2}}$,

where, $c_{0}=1 / 3$ and $c_{1}=2 / 3$ are called the ideal weights. $\epsilon=$ $10^{-40}$ is a small parameter introduced to avoid the denominator becoming zero.

The weight of the WENO-N3 [39] scheme is given as,

$\omega_{k}=\frac{\alpha_{k}}{\alpha_{0}+\alpha_{1}}, \alpha_{k}=c_{k} \cdot\left(1+\frac{\tau^{N}}{I S_{k}+\epsilon}\right)$,

where,

$\tau^{N}=\left|\frac{I S_{0}+I S_{1}}{2}-I S_{3}\right|$,

$I S_{3}=\frac{13}{12}\left(f_{i-1}-2 f_{i}+f_{i+1}\right)^{2}+\frac{1}{4}\left(f_{i-1}-f_{i+1}\right)^{2}$ is the second smoothness indicator of the fifth-order WENO scheme [3].

$I S_{k}$ in Eqs. (6)-(8) is called as the local smoothness indicator that measures the smoothness of numerical solution on the substencil $S_{k}$. The $I S_{k}$ of a third-order WENO scheme is usually calculated as follows $[2,3,36,39]$,

$\left\{\begin{array}{l}I S_{0}=\left(f_{i}-f_{i-1}\right)^{2} \\ I S_{1}=\left(f_{i+1}-f_{i}\right)^{2}\end{array}\right.$ 


\subsection{The WENO-MN3 scheme}

Since the WENO scheme with the indicators (9) is too dissipative and can not even reach second order(the order of $L_{\infty}$ error) convergence, Liu et al.[36] proposed the following indicators,

$\left\{\begin{array}{l}I S_{0}=\frac{1}{4}\left(\left|f_{i+1}-f_{i-1}\right|-\left|4 f_{i}-3 f_{i-1}-f_{i+1}\right|\right)^{2} \\ I S_{1}=\frac{1}{4}\left(\left|f_{i+1}-f_{i-1}\right|-\left|3 f_{i+1}+f_{i-1}-4 f_{i}\right|\right)^{2}\end{array}\right.$.

In monotonic smooth regions, there is $I S_{0}=I S_{1}$, hence, the weights in Eq. (6) calculated by the new indicators are exactly equal to the optimal weights, i.e., the fully third-order upwind scheme is recovered. Due to this property, the new scheme can greatly reduce the numerical dissipation and it is called as WENOMN3 in Liu and Shen [37]. Please find more details in Liu et al. [36], Liu and Shen [37].

\subsection{The HWENO-N3 scheme}

Considering the high resolution and low dissipation of the central scheme, it is a natural idea to conjugate the WNEO scheme with the central scheme to obtain a low dissipation shockcapturing scheme. In [39], Wu et al. designed a weighted hybrid scheme (HWENO-N3) of two sub-schemes: the third-order WENON3 scheme and the fourth-order central scheme,

$\hat{f}_{i+1 / 2}=\sigma_{i+1 / 2} \hat{f}_{i+1 / 2}^{C 4}+\left(1-\sigma_{i+1 / 2}\right) \hat{f}_{i+1 / 2}^{W E N O-N 3}$,

where, the weight function $\sigma_{i+1 / 2}$ is calculated as,

$\sigma_{i+1 / 2}=\frac{1}{2} \frac{\left.\tanh \left(3 \frac{r_{i+1 / 2}-r_{d}}{\max \left(r_{d}, r_{i+1 / 2}-r_{d}\right.}\right)\right)}{\tanh (3)}+\frac{1}{2}$,

and

$r_{i+1 / 2}=\min \left(r_{i}, r_{i+1}\right), r_{i}=\frac{\left|2 \delta_{i}^{+} \delta_{i}^{-}\right|+\epsilon}{\left(\delta_{i}^{+}\right)^{2}+\left(\delta_{i}^{-}\right)^{2}+\epsilon}$

where,

$\delta_{i}^{+}=f_{i+1}-f_{i}, \delta_{i}^{-}=f_{i}-f_{i-1}, \epsilon=\frac{0.9 r_{c}}{1-0.9 r_{c}} \xi^{2}$

The threshold values $r_{d}=0.25, \xi=10^{-10}$ and $r_{c}=0.5$ are suggested in $\mathrm{Wu}$ and Zhao [39]. Please refer to [35,39] for more information about the $r_{i+1 / 2}$ and $\sigma_{i+1 / 2}$.

The flux of the fourth-order central scheme(C4) is,

$\hat{f}_{i+1 / 2}^{C 4}=\frac{1}{12}\left(-f_{i-1}+7 f_{i}+7 f_{i+1}-f_{i+2}\right)$.

Although the HWENO-N3 scheme can decrease the numerical dissipation [39], as our results show, it generates spurious waves and asymmetric solutions near discontinuities.

\subsection{The S-HWMN3 scheme}

Although the WENO-MN3 scheme has less numerical dissipation than the WENO-JS3 and WENO-N3 schemes, the improvement of accuracy in $L_{\infty}$ norm of a smooth solution with critical points (e.g., the non-monotonic smooth regions) is still not remarkable [36]. As the analysis in Liu and Shen [37] showed, the existed third-order WENO schemes with the local smoothness indicators of Jiang and Shu[3] and Liu et al.[36] cannot achieve third-order convergence order at critical points, because these indicators cannot correctly treat the smooth three-point stencil containing a nonnodal critical point. Hence, Liu et al.[37] proposed a discontinuitydetecting method for a four-point stencil to distinguish those stencils containing a non-nodal critical point as follows,

$\left\{\begin{array}{l}\theta=\left|I C_{1}-I C_{2}\right| \geq \min \left(I C_{1}, I C_{2}\right), \\ \tau=\left|I S_{0}-I S_{1}\right| \geq \min \left(I S_{0}, I S_{1}\right)\end{array}\right.$ where, $I S_{0}, I S_{1}$ are the indicators of Eq. (10) used in the WENOMN3 scheme [36] and

$\left\{\begin{array}{l}I C_{1}=\left(f_{i-1}-2 f_{i}+f_{i+1}\right)^{2} \\ I C_{2}=\frac{1}{4}\left(\left|f_{i+2}-f_{i}\right|-\left|3 f_{i+2}+f_{i}-4 f_{i+1}\right|\right)^{2} .\end{array}\right.$

The stencil $S^{3}=(i-1, i, i+1)$ can be regarded as a discontinuous stencil, only if Eq. (16) is satisfied. Please find more details about the analysis of the discontinuity-detecting method in Liu and Shen [37]. Hence, a hybrid scheme of the WENO-MN3 scheme and the third-order upwind (U3) scheme can be designed as follows,

$\hat{f}_{i+1 / 2}= \begin{cases}\hat{f}_{i+1 / 2}^{W E N O M N 3}, & \text { if Eq. (16) is satisfied } \\ \hat{f}_{i+1 / 2}^{U 3}, & \text { otherwise. }\end{cases}$

where, $\hat{f}_{i+1 / 2}^{U 3}=c_{0} q_{0}+c_{1} q_{1}$. Since the WENO-MN3 scheme can recovery to third-order upwind scheme in monotonic smooth regions, a simplified hybrid scheme S-HWMN3 [37](combination of WENO-MN3 and U3) is suggested as follows,

$\hat{f}_{i+1 / 2}= \begin{cases}\hat{f}_{i+1 / 2}^{W E N O-M N 3}, & \text { if } \theta \geq \min \left(I C_{1}, I C_{2}\right) \\ \hat{f}_{i+1 / 2}^{U 3}, & \text { otherwise. }\end{cases}$

The numerical results [37] showed that the S-HWMN3 scheme can achieve third-order accuracy in smooth regions and keep the ENO property near discontinuities.

\section{The new hybrid WENO scheme: HWENO-U3C4}

In this section, we propose a two-step method for constructing a new hybrid central WENO scheme.

As the results in Refs. $[34,35,39]$ and our numerical tests show, a direct switch from ENO or WENO schemes to compact or central schemes may generate spurious waves. While, the results in Liu and Shen [37], Li and Qiu [46], 47] showed that, the switch from WENO schemes to upwind schemes behaves quite well for all the tested cases near shock regions. Hence, based on the S-HWMN3 scheme, a new hybrid scheme is proposed as following,

$\hat{f}_{i+1 / 2}= \begin{cases}\hat{f}_{i+1 / 2}^{W E N O-M N 3}, & \text { if Eq. (16) is satisfied } \\ \hat{f}_{i+1 / 2}^{W U 3 C 4}, & \text { otherwise. }\end{cases}$

where, the sub-scheme $\hat{f}_{i+1 / 2}^{W U 3 C 4}$ (denoted as WU3C4) is a weighted scheme of the third-order upwind scheme(U3) and the fourthorder central scheme(C4).

\subsection{The WU3C4 sub-scheme}

The numerical flux $\hat{f}_{i+1 / 2}^{W U 3 C 4}$ is calculated as,

$\hat{f}_{i+1 / 2}^{W U 3 C 4}=\sigma \hat{f}_{i+1 / 2}^{U 3}+(1-\sigma) \hat{f}_{i+1 / 2}^{C 4}$,

where, $\sigma$ is a weight function introduced to avoid an abrupt switch from WENO-MN3 scheme to the central scheme and apply the fourth-order central scheme (C4) in smooth regions as possible. Hence, the weight function $\sigma$ should approach to 0 for a smooth stencil $S^{4}=(i-1, i, i+1, i+2)$, and to 1 if there is a discontinuity located in $(i+1, i+2)$.

Inspired by the work of Shen et al.[14], Peng et al.[48] and Liu et al.[12], a simple weight function is designed as follows,

$\sigma=\frac{\left|\beta_{1}-\beta_{2}\right|}{\max \left(\beta_{1}, \beta_{2}\right)+\epsilon}$

where, $\epsilon=10^{-40}$ is introduced to avoid the denominator to become zero, and

$\left\{\begin{array}{l}\beta_{1}=\frac{13}{12}\left(f_{i-1}-2 f_{i}+f_{i+1}\right)^{2}+\frac{1}{4}\left(f_{i-1}-f_{i+1}\right)^{2}, \\ \beta_{2}=\frac{13}{12}\left(f_{i}-2 f_{i+1}+f_{i+2}\right)^{2}+\frac{1}{4}\left(3 f_{i}-4 f_{i+1}+f_{i+2}\right)^{2},\end{array}\right.$ 
are the two local smoothness indicators of the fifth-order WENO scheme [3].

For a smooth solution, as the Taylor expansion analysis in Liu et al. [12], Shen et al. [14], Peng and Shen [48] showed, there is

$\sigma=\frac{f_{i}^{\prime} f_{i}^{\prime \prime \prime} \Delta x^{4}+A \Delta x^{5}}{f_{i}^{\prime \prime 2} \Delta x^{2}+B_{k} \Delta x^{4}}= \begin{cases}O\left(\Delta x^{2}\right), & \text { if } f_{i}^{\prime} \neq 0, \\ O(\Delta x), & f_{i}^{\prime}=0 .\end{cases}$

where, $A$ and $B_{k}, k=1,2$ are non-zero constant. Eq. (24) means that, for a smooth stencil $S^{4}, \sigma$ is a small magnitude.

If the sub-stencil $(i-1, i)$ or $(i, i+1)$ contains a discontinuity, then the WENO-MN3 scheme is used according to the detecting method in Eq. (16); otherwise the stencil $(i-1, i, i+1)$ is smooth, and if the sub-stencil $(i+1, i+2)$ contains a discontinuity, there should be

$\beta_{2} \gg \beta_{1}$.

which means, $\sigma$ approaches to 1 , and hence the WU3C4 scheme is dominated by the U3 scheme.

\subsection{The behavior near discontinuities}

This subsection compares the behavior of different schemes near discontinuities. In order to show the deficiency of the hybrid scheme, in which the fourth-order central scheme is directly switched, in this subsection, a hybrid central WENO scheme(denoted as HWENO-C4) is designed as follows,

$\hat{f}_{i+1 / 2}= \begin{cases}\hat{f}_{i+1 / 2}^{W E N O M N 3}, & \text { if } S^{4}=(i-1, i, i+1, i+2) \\ \hat{f}_{i+1 / 2}^{C 4}, & \text { is a discontinuous stencil, }\end{cases}$

In this paper, a detecting-method is used to determine whether $S^{4}$ contains a continuity or not, i.e., if

$\begin{cases}\left|I S_{0}-I S_{1}\right|_{(i-1, i, i+1)} & \geq \min \left(I S_{0}, I S_{1}\right)_{(i-1, i, i+1)} \\ \left|I S_{0}-I S_{1}\right|_{(i, i+1, i+2)} & \geq \operatorname{or}\end{cases}$

is satisfied, then $S^{4}$ can be regarded as a discontinuous stencil. Where, $\left(I S_{0}, I S_{1}\right)_{(i-1, i, i+1)}$ are the smoothness indicators on the stencil $(i-1, i, i+1)$ and calculated by Eq. (10). The first condition (Eq. (27)) indicates that $S_{1}^{3}=(i-1, i, i+1)$ may contain a discontinuity, otherwise, we can apply the Taylor expansion, and there is $\left|I S_{0}-I S_{1}\right|<\min \left(I S_{0}-I S_{1}\right)$ (Please see Ref. [36,37,49] for more information). So does the second condition.

The linear advection equation Eq. (29) with the initial condition,

$u_{0}(x)= \begin{cases}1, & -0.5 \leq x \leq 0.5 \\ 0, & \text { else }\end{cases}$

is solved to demonstrate the behaviors of different schemes.

This simple case represents the propagation of a square wave, its solutions computed by the WENO-MN3, HWENO-N3, HWENOC4 and HWENO-U3C4 schemes at $t=8$ with $N=80$ are plotted in Figs. 1-4 are the pointwise errors. It can be seen that, the HWENO-N3 scheme behaves similar with the HWENO-C4 scheme, although both of them can preserve the ENO property well, they generate apparently asymmetric solutions near the discontinuities. And the new scheme HWENO-U3C4 resolves the discontinuous problem quite well. The numerical results show that, the direct switch from the third-order WENO scheme to the fourth-order central scheme may generate spurious waves near the discontinuities. Since HWENO-N3 and HWENO-C4 have the similar deficiency near discontinuities, in the rest of this paper, the results of HWENO-C4 are no longer presented and discussed.

Numerical stability of a method is a necessary property for its applications in time integration problems. The stability analysis of fifth-order WENO scheme can be found in Wang and Spiteri [50], Motamed et al. [51], Hermes et al. [52]. However, as

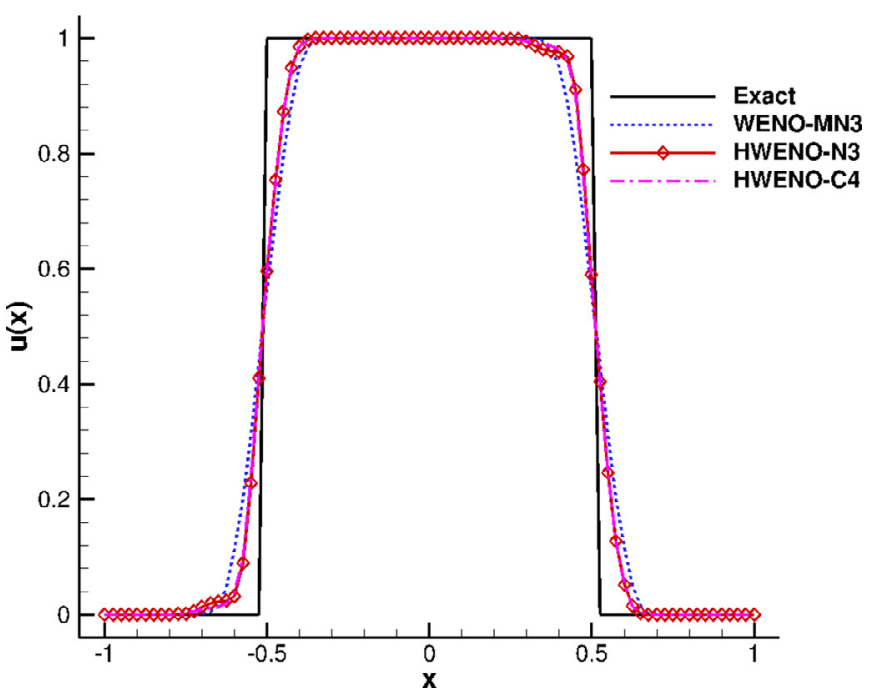

Fig. 1. Solutions of a square wave computed by the WENO-MN3, HWENO-N3 and HWENO-C4 schemes with $N=80$ at $t=8$.

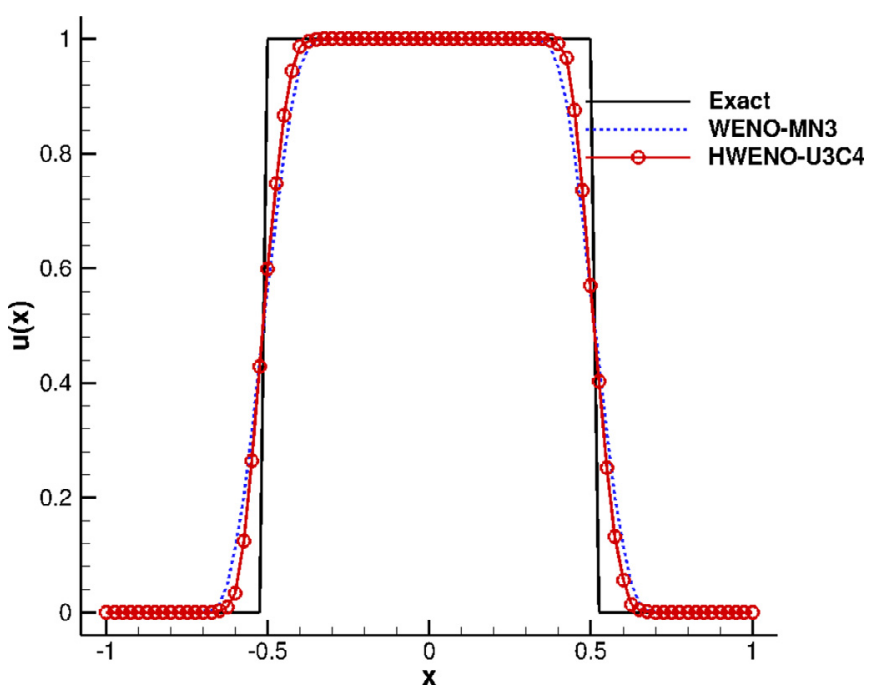

Fig. 2. Solutions of a square wave computed by the WENO-MN3 and HWENO-U3C4 schemes with $N=80$ at $t=8$.

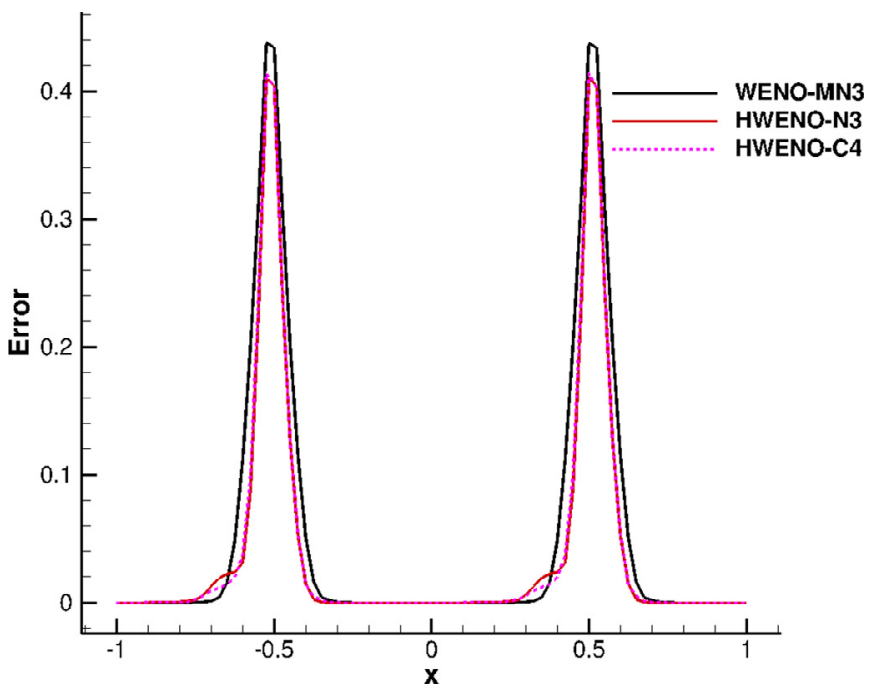

Fig. 3. Pointwise error of the WENO-MN3, HWENO-N3 and HWENO-C4 schemes in Fig. 1. 


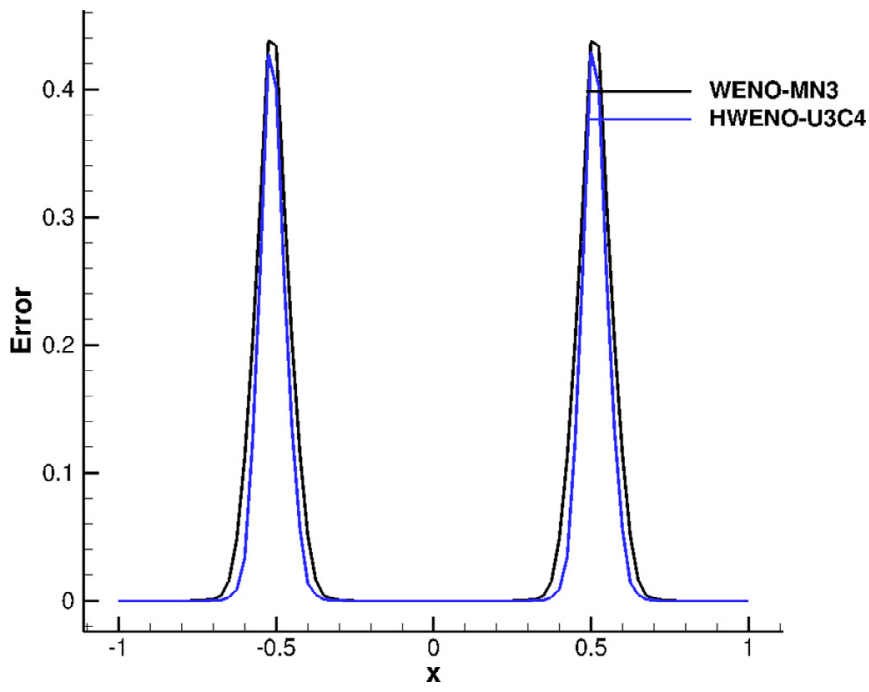

Fig. 4. Pointwise error of the WENO-MN3 and HWENO-U3C4 schemes in Fig. 2.

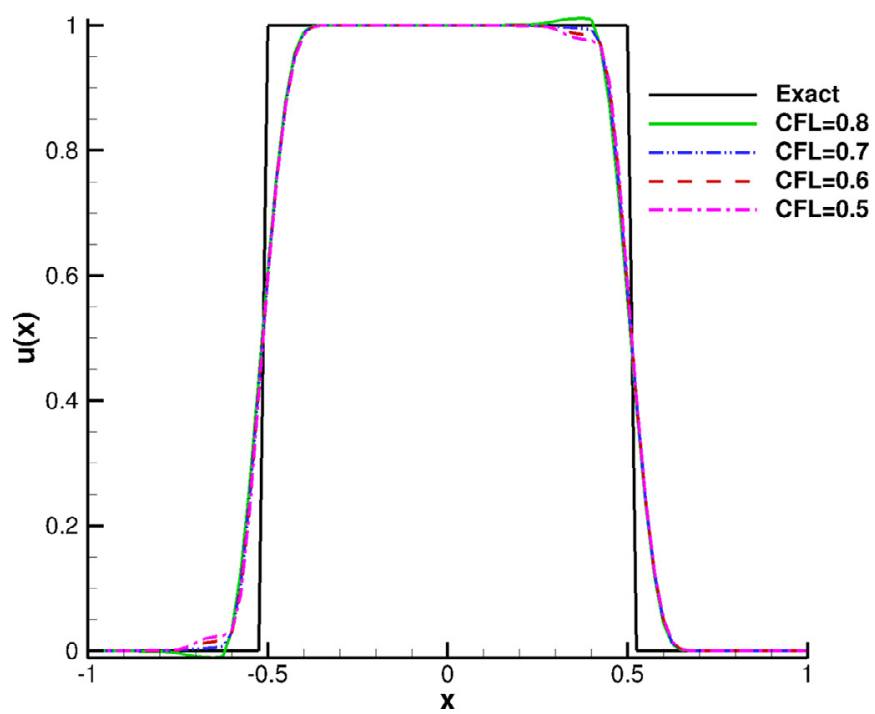

Fig. 5. Solutions computed by the HWENO-N3 scheme with different CFL numbers at $N=80$ and $t=8$.

pointed out by Wang and Spiteri[50], the von Neumann stability analysis needs to have a known stencil. Since the present scheme (HWENO-U3C4) is a hybrid scheme, in which, one candidate scheme uses a three-point stencil and the other one uses a four-point stencil, it is difficult to do the linear stability analysis. Here, we solve the advection equation Eq. (29) with the initial condition of Eq. (28) and different CFL numbers to compare the stability of different schemes. The time integration method is the thirdorder TVD Runge-Kutta method [53]. Figs. 5-7 show that, for a discontinuous solution, HWENO-U3C4 and HWENO-N3 may generate unstable solutions(undershoot and overshoot) if $C F L>0.7$, while WENO-MN3 can obtain a good solution even CFL number takes 1.0. Hence, WENO-MN3 is more stable than the two hybrid schemes. Applying hybrid scheme requires a relatively strict restriction on time step.

\section{Numerical examples}

In this section, several linear advection problems, one- and twodimensional Euler problems are calculated to test the performance of the new scheme. The time derivative is approximated with the third-order TVD Runge-Kutta method [53].

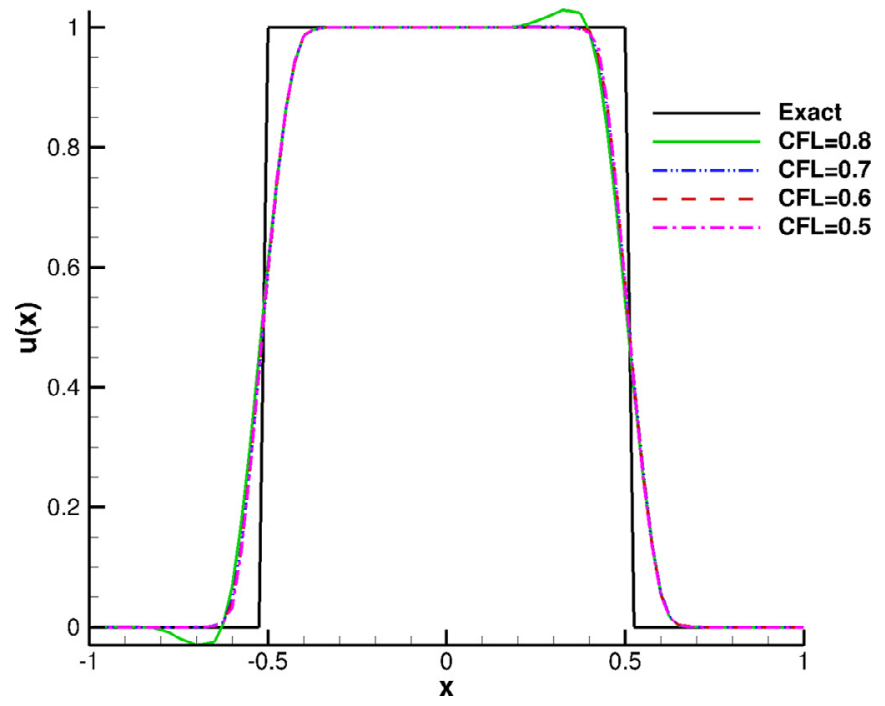

Fig. 6. Solutions computed by the HWENO-U3C4 scheme with different CFL numbers at $N=80$ and $t=8$.

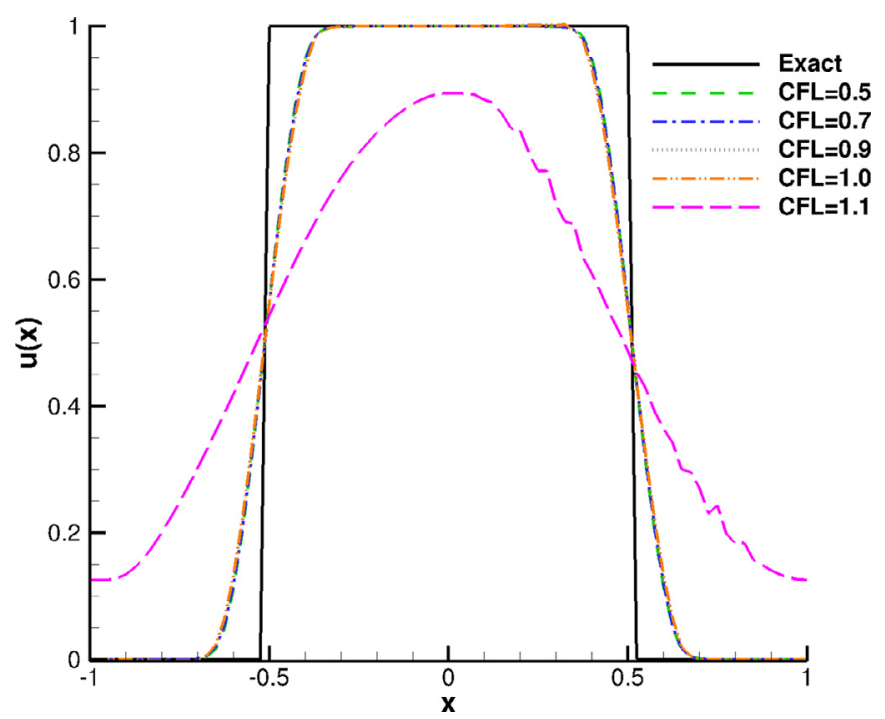

Fig. 7. Solutions computed by the WENO-MN3 scheme with different CFL numbers at $N=80$ and $t=8$.

\subsection{Linear advection problems}

In the following we test the schemes on the linear advection equation,

$\begin{cases}\frac{\partial u}{\partial t}+\frac{\partial u}{\partial x}=0, & x_{0} \leqslant x \leqslant x_{1}, \\ u(x, t=0)=u_{0}(x), & \text { periodic boundary. }\end{cases}$

The exact solution of Eq. (29) is given by,

$u(x, t)=u_{0}(x-t, t)$.

4.1.1. Case 1

$u_{0}(x)=\sin \left(\pi x-\frac{\sin \pi x}{\pi}\right),-1 \leq x \leq 1$.

This particular initial condition [6] has two critical points at which $f^{\prime}=0$ and $f^{\prime \prime} \neq 0$ and is often used to test the accuracy of a scheme. The time step is chosen to be $\Delta t=8 \Delta x^{5 / 3}$ in order that 
Table 1

Convergence rate in $L_{1}$ norm.

\begin{tabular}{|c|c|c|c|c|c|c|c|c|c|c|c|c|}
\hline \multirow[t]{2}{*}{$N$} & \multicolumn{2}{|l|}{ WENO-JS3 } & \multicolumn{2}{|c|}{ WENO-MN3 } & \multicolumn{2}{|l|}{ U3 } & \multicolumn{2}{|l|}{ S-HWMN3 } & \multicolumn{2}{|l|}{ C4 } & \multicolumn{2}{|c|}{ HWENO-U3C4 } \\
\hline & error & order & error & order & error & order & error & order & error & order & error & order \\
\hline 50 & $8.160 \mathrm{E}-2$ & & $1.347 \mathrm{E}-2$ & & $4.590 \mathrm{E}-3$ & & $4.590 \mathrm{E}-3$ & & $1.336 \mathrm{E}-3$ & & $1.512 \mathrm{E}-3$ & \\
\hline 100 & $1.425 \mathrm{E}-2$ & 2.518 & $4.647 \mathrm{E}-3$ & 1.536 & $4.597 \mathrm{E}-4$ & 3.32 & $4.597 \mathrm{E}-4$ & 3.32 & $4.701 \mathrm{E}-5$ & 4.83 & $5.511 \mathrm{E}-5$ & 4.78 \\
\hline 200 & $3.480 \mathrm{E}-3$ & 2.033 & $9.453 \mathrm{E}-4$ & 2.298 & $5.354 \mathrm{E}-5$ & 3.10 & $5.354 \mathrm{E}-5$ & 3.10 & $1.895 \mathrm{E}-6$ & 4.63 & $2.346 \mathrm{E}-6$ & 4.55 \\
\hline 400 & $8.160 \mathrm{E}-4$ & 2.092 & $1.768 \mathrm{E}-4$ & 2.418 & $6.567 \mathrm{E}-6$ & 3.03 & $6.567 \mathrm{E}-6$ & 3.03 & $9.508 \mathrm{E}-8$ & 4.32 & $1.253 \mathrm{E}-7$ & 4.23 \\
\hline 800 & $1.860 \mathrm{E}-4$ & 2.133 & $3.242 \mathrm{E}-5$ & 2.448 & $8.170 \mathrm{E}-7$ & 3.00 & $8.170 \mathrm{E}-7$ & 3.00 & $5.515 \mathrm{E}-9$ & 4.18 & $7.692 \mathrm{E}-9$ & 4.03 \\
\hline
\end{tabular}

Table 2

Convergence rate in $L_{\infty}$ norm.

\begin{tabular}{|c|c|c|c|c|c|c|c|c|c|c|c|c|}
\hline \multirow[t]{2}{*}{$N$} & \multicolumn{2}{|l|}{ WENO-JS3 } & \multicolumn{2}{|c|}{ WENO-MN3 } & \multicolumn{2}{|l|}{ U3 } & \multicolumn{2}{|l|}{ S-HWMN3 } & \multicolumn{2}{|l|}{ C4 } & \multicolumn{2}{|c|}{ HWENO-U3C4 } \\
\hline & error & order & error & order & error & order & error & order & error & order & error & order \\
\hline 50 & $9.742 \mathrm{E}-2$ & & $3.399 \mathrm{E}-2$ & & $5.674 \mathrm{E}-3$ & & $5.674 \mathrm{E}-3$ & & $1.661 \mathrm{E}-3$ & & $1.748 \mathrm{E}-3$ & \\
\hline 100 & $3.119 \mathrm{E}-2$ & 1.643 & $1.494 \mathrm{E}-2$ & 1.186 & $5.766 \mathrm{E}-4$ & 3.30 & $5.766 \mathrm{E}-4$ & 3.30 & $5.992 \mathrm{E}-5$ & 4.79 & $9.528 \mathrm{E}-5$ & 4.20 \\
\hline 200 & $1.207 \mathrm{E}-3$ & 1.370 & $5.083 \mathrm{E}-3$ & 1.556 & $6.732 \mathrm{E}-5$ & 3.10 & $6.732 \mathrm{E}-5$ & 3.10 & $2.562 \mathrm{E}-6$ & 4.55 & $7.324 \mathrm{E}-6$ & 3.70 \\
\hline 400 & $4.561 \mathrm{E}-3$ & 1.404 & $1.684 \mathrm{E}-3$ & 1.594 & $8.263 \mathrm{E}-6$ & 3.03 & $8.263 \mathrm{E}-6$ & 3.03 & $1.350 \mathrm{E}-7$ & 4.25 & $6.369 E-7$ & 3.52 \\
\hline 800 & $1.710 \mathrm{E}-3$ & 1.415 & $5.467 \mathrm{E}-4$ & 1.623 & $1.028 \mathrm{E}-6$ & 3.00 & $1.028 \mathrm{E}-6$ & 3.00 & $7.992 \mathrm{E}-9$ & 4.08 & $6.019 \mathrm{E}-8$ & 3.40 \\
\hline
\end{tabular}

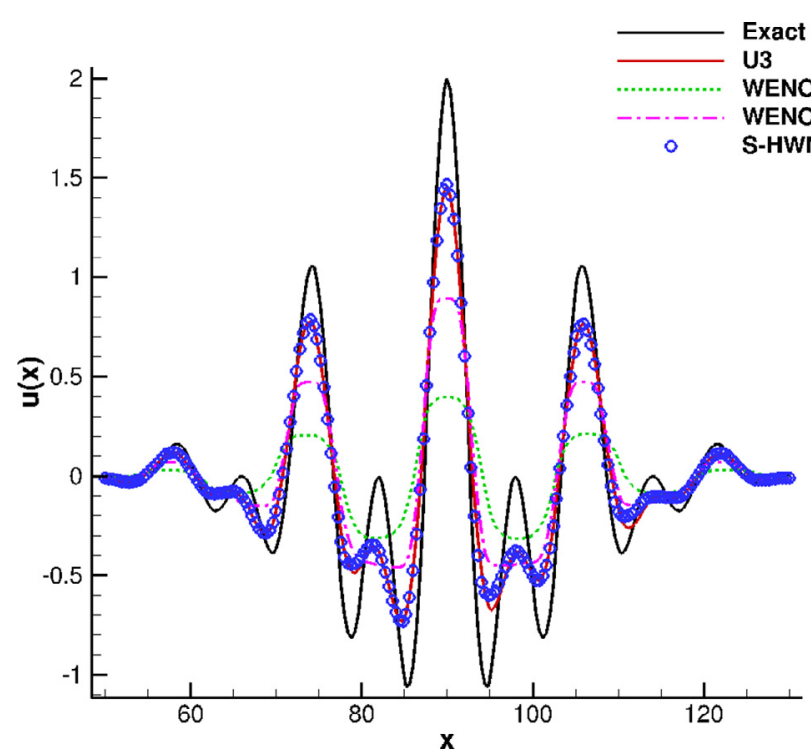

Fig. 8. Solutions of Case 2 computed by the U3, WENO-JS3, WENO-MN3 and SHWMN3 schemes with $N=200$ at $t=320$.

the error for the overall scheme is a measure of the spatial convergence only [6]. The errors and the convergence order in $L_{1}$ and $L_{\infty}$ norm of the WENO-JS3, WENO-MN3, U3, S-HWMN3, C4 and HWENO-U3C4 schemes at $t=2$ are given in Tables 1 and 2. As we can see, the S-HWMN3 scheme recovers to the U3 scheme, while, the order in $L_{1}$ of the HWENO-U3C4 scheme achieves fourth-order. Although the order in $L_{\infty}$ does not achieve the expected fourth order, its errors are much less than those of U3.

\subsubsection{Case 2}

$u_{0}(x)=e^{-(x-90)^{2} / 400}\left(\cos \left(\frac{\pi}{8}(x-90)\right)+\cos \left(\frac{\pi}{4}(x-90)\right)\right)$,

$$
50 \leq x \leq 130 .
$$

This case is used to test the performance of different schemes for a smooth solution containing several critical points [54]. Figs. 8 and 9 show the numerical solutions with grid number $N=200$ and time step $\Delta t=\Delta x / 2$ at $t=320$. As shown in these figures, the results of the C4 and HWENO-U3C4 schemes are almost the same.

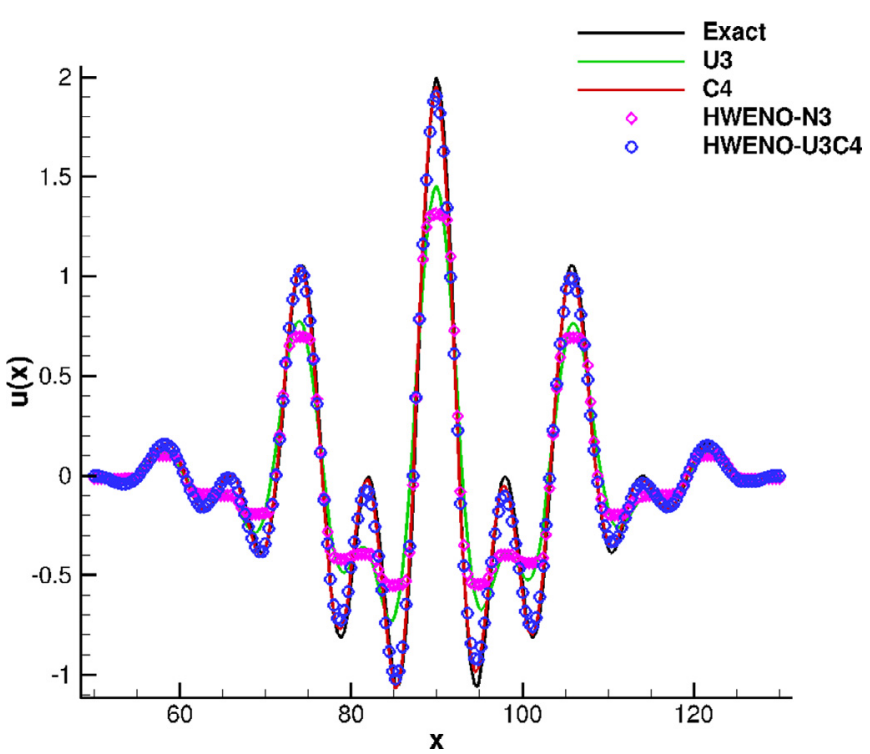

Fig. 9. Solutions of Case 2 computed by the U3, C4, HWENO-N3 and HWENO-U3C4 schemes with $N=200$ at $t=320$.

4.1.3. Case 3

$u_{0}(x)=\left\{\begin{array}{l}\frac{1}{6}(G(x, \beta, z-\delta)+G(x, \beta, z+\delta)+4 G(x, \beta, z)), \\ \quad-0.8 \leqslant x<-0.6, \\ 1, \quad-0.4 \leqslant x<-0.2, \\ 1-|10(x-0.1)|, \quad 0 \leqslant x<0.2, \\ \frac{1}{6}(F(x, \alpha, a-\delta)+F(x, \alpha, a+\delta)+4 F(x, \alpha, a)), \\ \quad 0.4 \leqslant x<0.6, \\ 0, \quad \text { otherwise, }\end{array}\right.$

$G(x, \beta, z)=e^{-\beta(x-z)^{2}}, F(x, \beta, a)=\sqrt{\max \left(1-\alpha^{2}(x-a)^{2}, 0\right)}$.

This is a classical testing case, which contains a Gaussian, a triangle, a square-wave and a semi-ellipse. It is often used to test the dissipation of high-order schemes. The constants are $z=-0.7, \delta=$ $0.005, \beta=\log (2) /\left(36 \delta^{2}\right), \quad a=0.5$ and $\alpha=10$. It is solved with $N=200$ and time step $\Delta t=\Delta x / 2$. Figs. 10-13 display the results at $t=2$ and $t=20$, respectively. It can be seen that, after a long time period, the HWENO-U3C4 scheme still resolves all the 


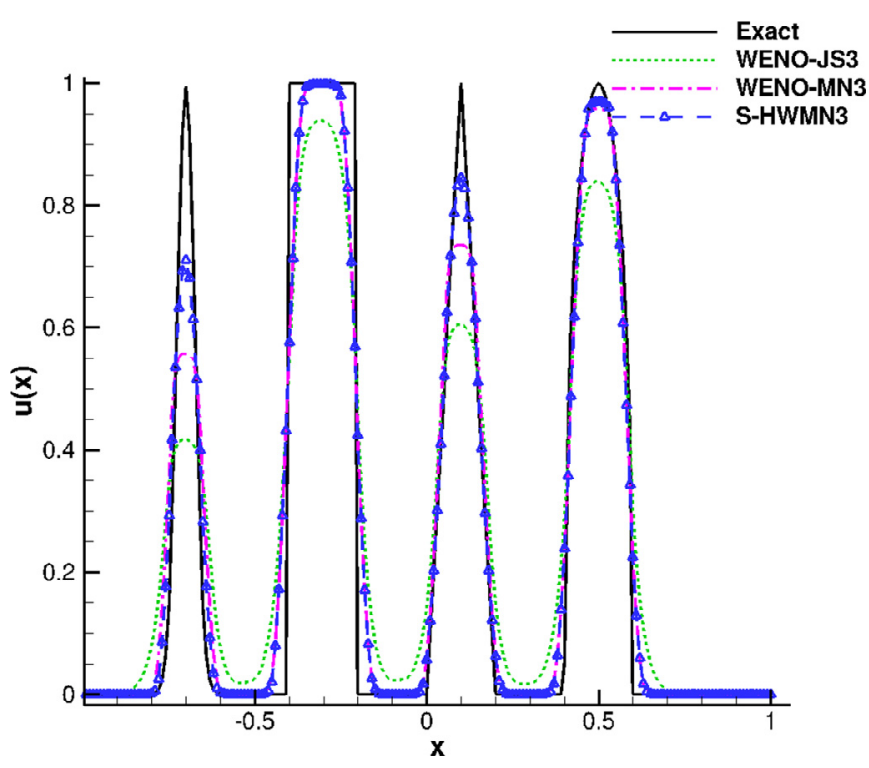

Fig. 10. Solutions of Case 3 computed by the WENO-JS3, WENO-MN3 and SHWMN3 schemes with $N=200$ at $t=2$.

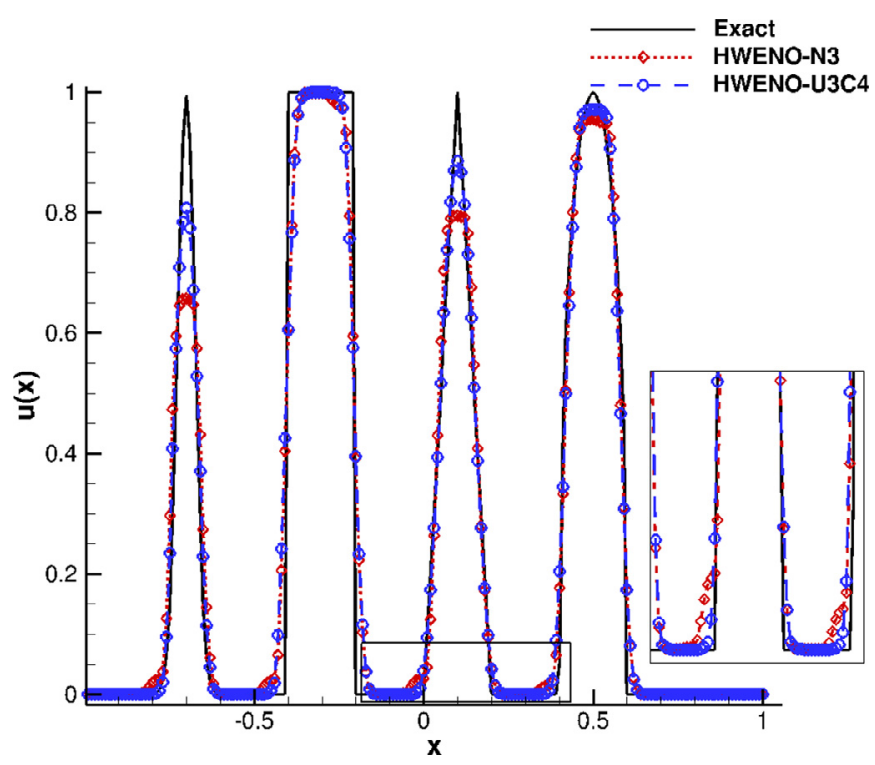

Fig. 11. Solutions of Case 3 computed by the HWENO-N3 and HWENO-U3C4 schemes with $N=200$ at $t=2$

four waves quite well, but the HWENO-N3 scheme generates more serious asymmetric solutions near discontinuities. The HWENOU3C4 scheme shows less numerical dissipation than the HWENON3 scheme [39].

4.1.4. Case 4

$u_{0}(x)= \begin{cases}-x \sin \left(\frac{3 \pi}{2} x^{2}\right), & \text { if }-1 \leq x \leq-\frac{1}{3} \\ |\sin (2 \pi x)|, & \text { if }-\frac{1}{3}<x \leq \frac{1}{3}, \\ 2 x-1-\frac{1}{6} \sin (3 \pi x), & \text { if } \frac{1}{3} \leq x \leq 1\end{cases}$

This initial solution [8] consists of contact discontinuities, corner singularities and smooth areas. The solutions at $t=41$ with $N=400$ and time step $\Delta t=\Delta x / 2$ are plotted in Figs. 14 and 15 . As shown in Fig. 15, even in the smooth regions, the HWENO$\mathrm{N} 3$ scheme also generates spurious waves. One possible reason is that, certain smooth regions may be treated as non-smooth regions

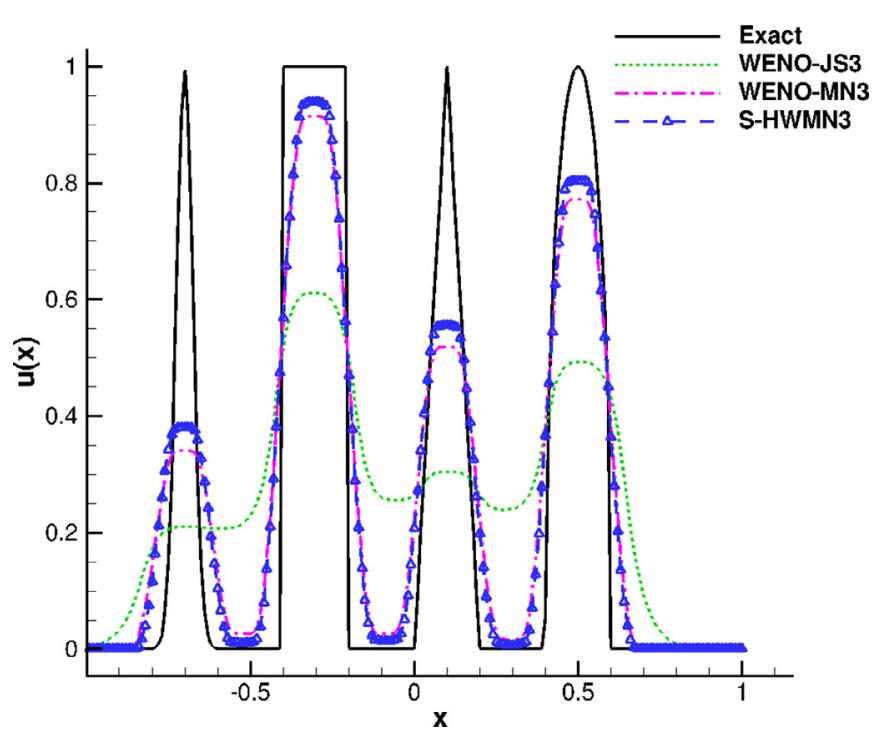

Fig. 12. Solutions of Case 3 computed by the WENO-JS3, WENO-MN3 and SHWMN3 schemes with $N=200$ at $t=20$.

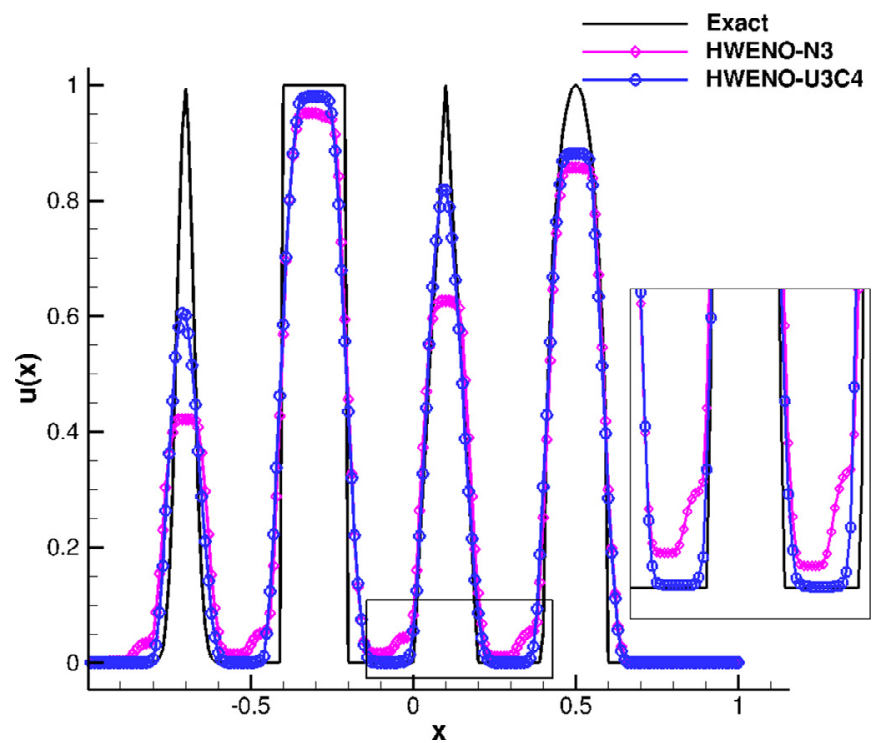

Fig. 13. Solutions of Case 3 computed by the HWENO-N3 and HWENO-U3C4 schemes with $N=200$ at $t=20$.

by the weight function in Eq. (12). As we can see in Fig. 14, the HWENO-U3C4 scheme works best.

\subsection{One-dimensional Euler problems}

The governing equations are as follows,

$\frac{\partial U}{\partial t}+\frac{\partial F}{\partial x}=0$,

where $U=(\rho, \rho u, E)^{T}, \quad F(U)=\left(\rho u, \rho u^{2}+p, u(E+p)\right)^{T}, \quad \rho, \quad u$, $E$ and $p$ are density, velocity, total energy and pressure, respectively. For the ideal gas, $E=\frac{p}{\gamma-1}+\frac{1}{2} \rho u^{2}$, where $\gamma=1.4$ is the ratio of specific heat. Time step is taken as,

$\Delta t=\frac{\sigma \Delta x}{\max _{i}\left(\left|u_{i}\right|+c_{i}\right)}$,

where, $\sigma=0.5$ is CFL number. $c$ is the speed of sound and given by $c=\sqrt{\gamma p / \rho}$. Three shock-tube problems [7,53] are tested. The global LF splitting method $[26,55]$ is used and all the reference 


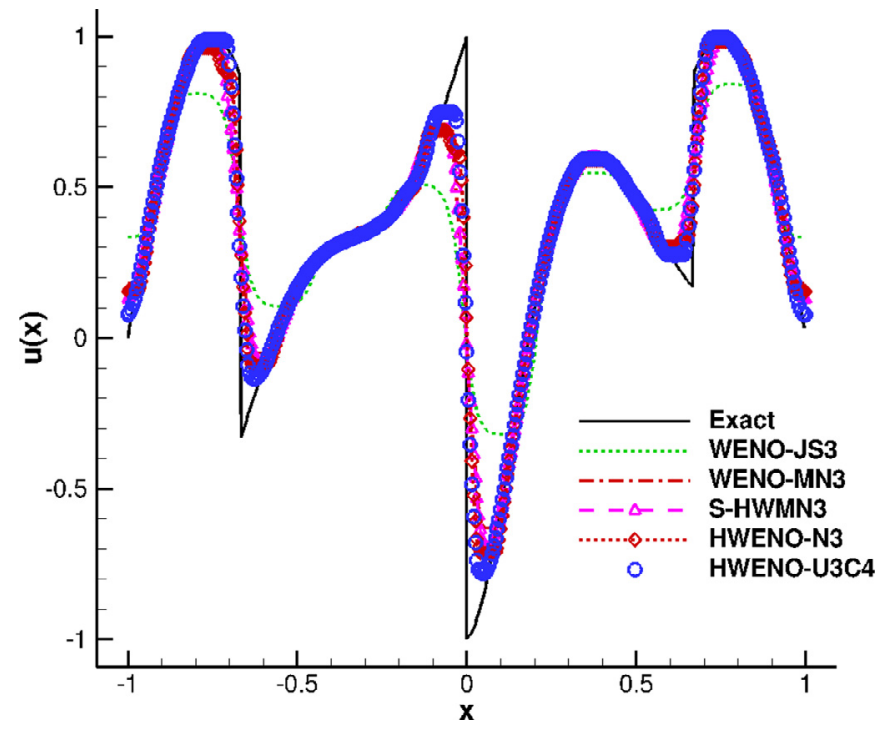

Fig. 14. Solutions of Case 4 computed by the WENO-JS3, WENO-MN3, S-HWMN3, HWENO-N3 and HWENO-U3C4 schemes with $N=400$ at $t=41$.

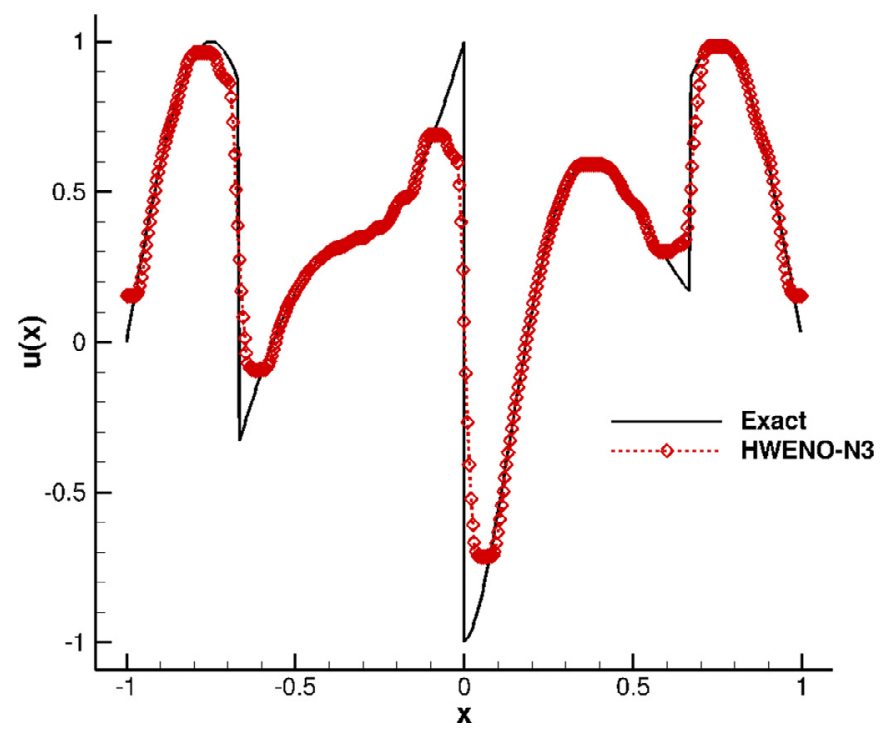

Fig. 15. Solutions of Case 4 computed by the HWENO-N3 scheme with $N=400$ at $t=41$.

solutions are obtained by the WENO-MN3 scheme with a grid of 5000 .

\subsubsection{Case 5}

The first 1-D case is the Shu-Osher problem [53] with the initial condition,

$$
(\rho, u, p)= \begin{cases}(3.857143,2.629369,31 / 3) & -5 \leq x<-4 \\ (1+0.2 \sin (5 x), 0,1) & -4 \leq x \leq 5\end{cases}
$$

Figs. 16 and 17 give the distributions of density at $t=1.8$ by using $N=600$. As these figures show, the HWENO-U3C4 scheme is less dissipative than the S-HWMN3 and HWENO-N3 schemes.

From Fig. 17, the results for the Shu-Osher problem appear some dispersion error. In order to investigate this phenomenon, we apply the characteristic Roe-type flux [55] method combined with different WENO reconstructions, and find the dispersion error can be alleviated. For simplicity, only the results of HWENO-U3C4 are given in Fig. 18.

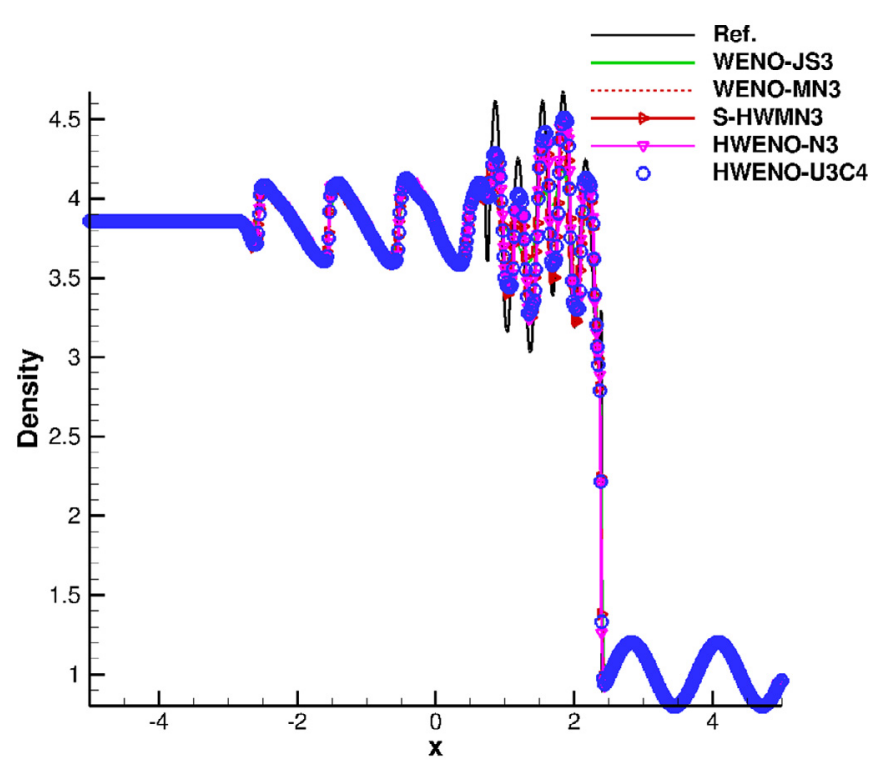

Fig. 16. Solutions of Case 5 computed by the WENO-JS3, WENO-MN3, S-HWMN3, HWENO-N3 and HWENO-U3C4 schemes with $N=600$ at $t=1.8$.

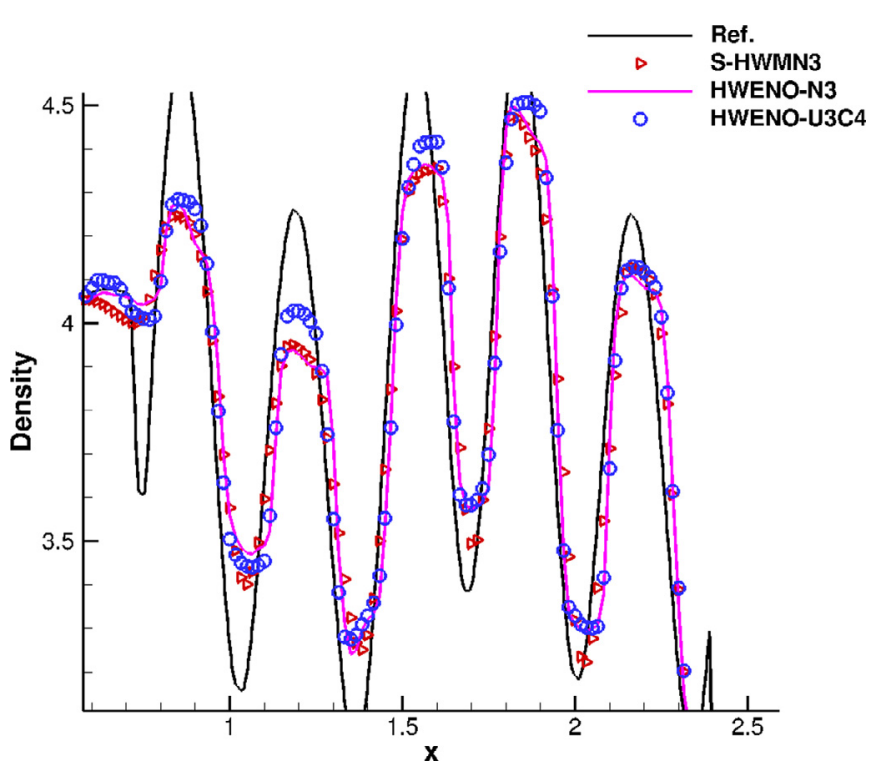

Fig. 17. Enlarged plot of the S-HWMN3, HWENO-N3 and HWENO-U3C4 schemes in Fig. 16.

\subsubsection{Case 6}

In order to compare various schemes and test their dispersion errors, the second case with following initial conditions is calculated,

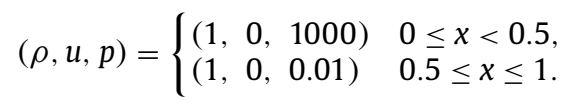

Figs. 19 and 20 give the results with $N=200$ and $t=0.012$. Similar as the Shu-Osher problem, the dispersion errors are also generated, and they can be alleviated by using the characteristic Roe-type [55] flux method.

Since the third-order upstream scheme has larger dissipation and dispersion errors than the fifth-order upstream scheme, the dispersive phenomenon of the former is more apparent than that of the latter. And hence, these schemes related to the third-order upstream scheme may generate some dispersion errors. But with a 


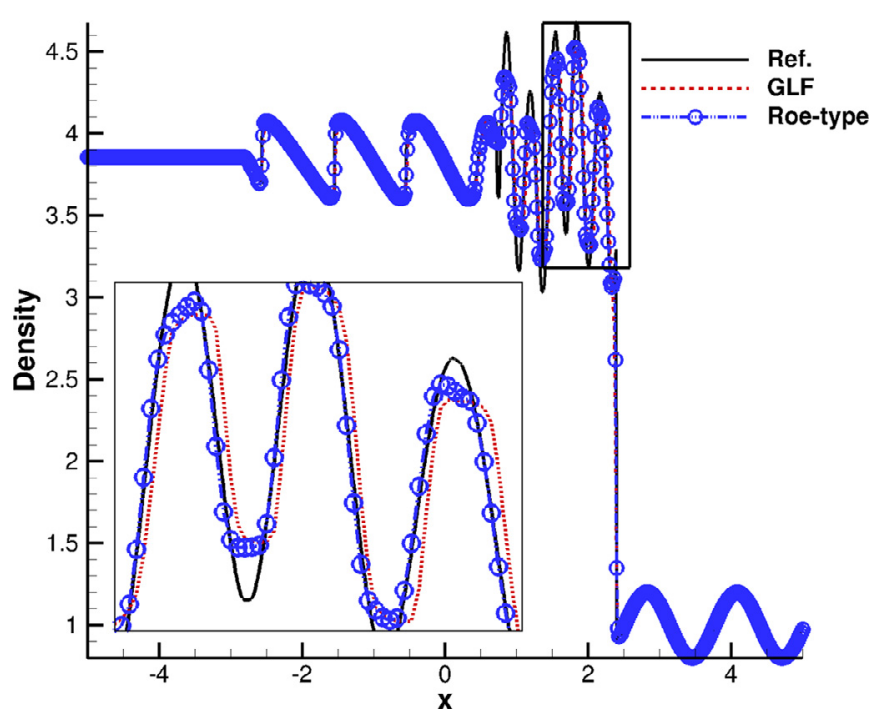

Fig. 18. Solutions of Case 5 computed by the HWENO-U3C4 scheme with GLF and Roe-type flux at $N=600$.

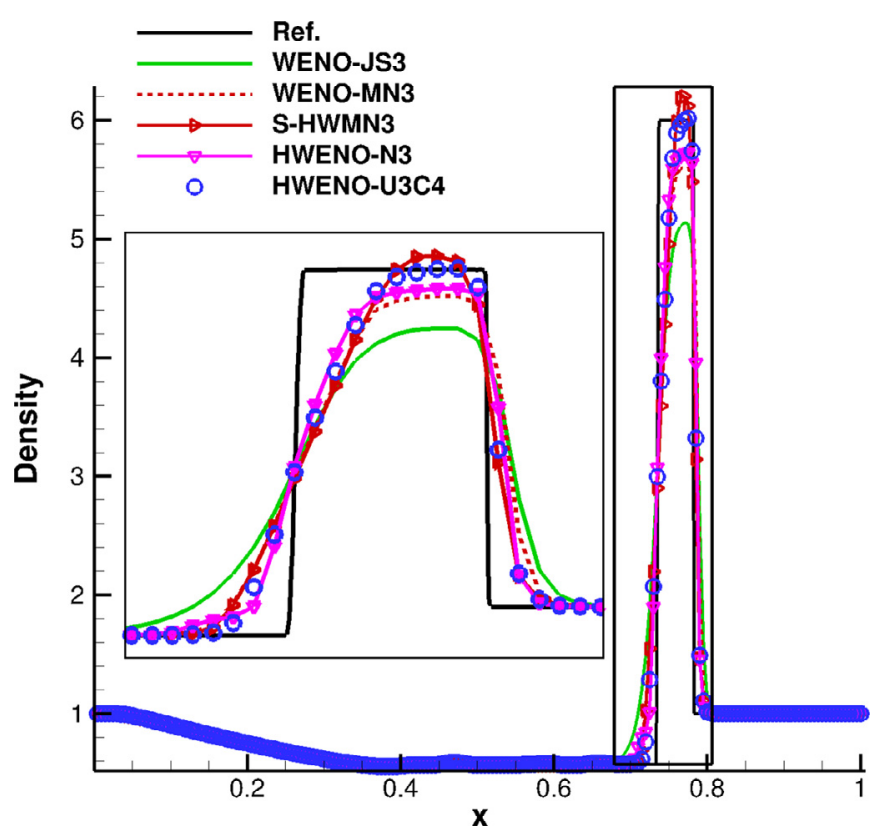

Fig. 19. Solutions of Case 6 computed by the WENO-JS3, WENO-MN3, S-HWMN3 and HWENO-U3C4 schemes with LF flux, $N=200, t=0.012$.

high resolution Riemann solver, the dispersion errors can be alleviated.

\subsubsection{Case 7}

The third case is the interactive blast waves problem [7] with the initial condition,

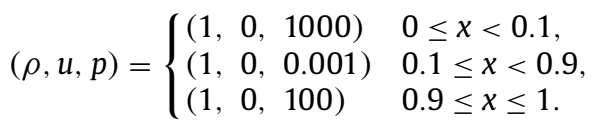

First, we test these schemes with component-wise reconstruction method, the numerical results at $t=0.038$ with $N=600$ are presented in Fig. 21. In this case, due to the small value $(0.001)$ of pressure, a small overshoot may generate negative pressure and make the computation breakdown, and hence no results can be obtained by the HWENO-N3 scheme. In order to make sure of its behavior, these schemes with the characteristic-wise reconstruction method [26,55] are also tested, and the solutions are

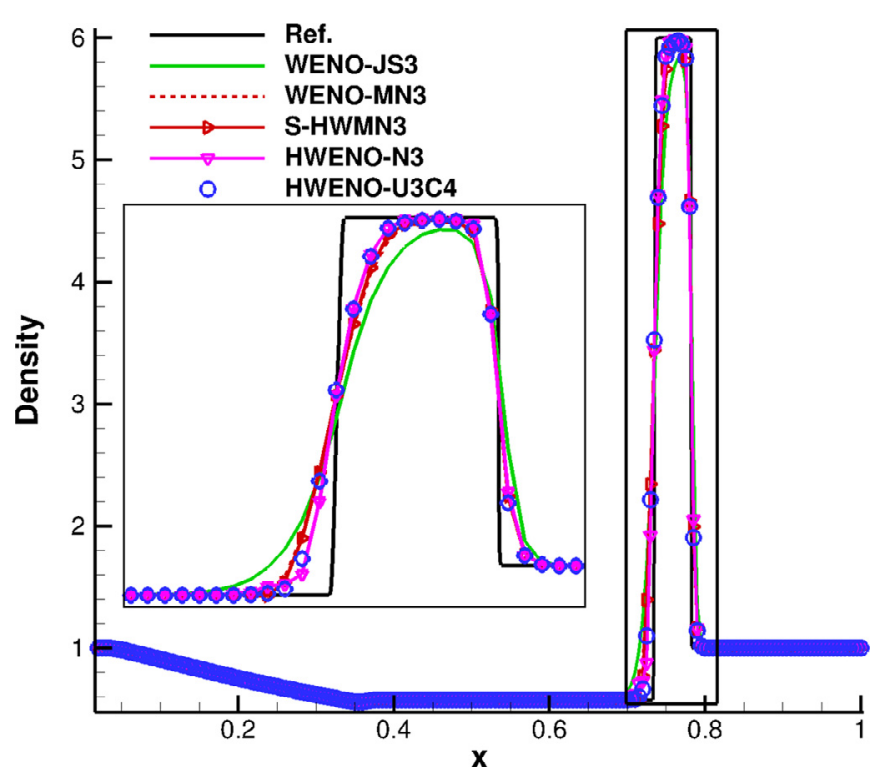

Fig. 20. Solutions of Case 6 computed by the WENO-JS3, WENO-MN3, S-HWMN3, HWENO-N3 and HWENO-U3C4 schemes with Roe-type flux, $N=200, t=0.012$.

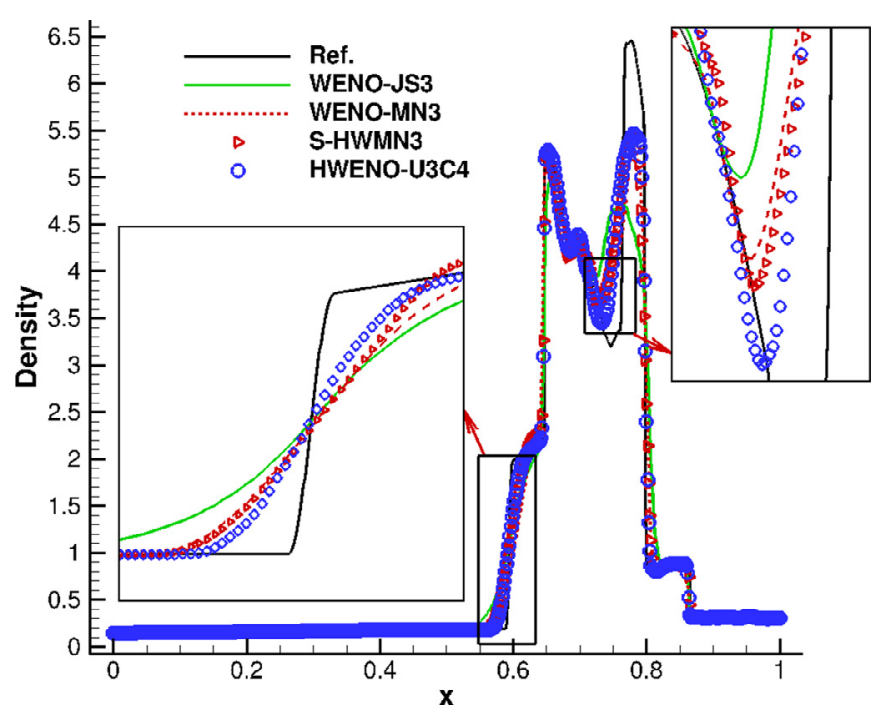

Fig. 21. Solutions of Case 7 computed by the WENO-JS3, WENO-MN3, S-HWMN3 and HWENO-U3C4 schemes with component-wise reconstruction method.

given in Fig. 22. Numerical results show that, the HWENO-U3C4 scheme performs very well for both reconstruction methods. Even the characteristic-wise reconstruction method is implemented, the HWENO-N3 scheme still generates spurious waves near shocks.

\subsection{Two-dimensional Euler problems}

The governing equation is the two-dimensional Euler equations,

$\frac{\partial U}{\partial t}+\frac{\partial F}{\partial x}+\frac{\partial G}{\partial y}=0$

where the conservative variables $U$, the inviscid flux vectors $F$ and $G$ are,

$$
U=\left[\begin{array}{c}
\rho \\
\rho u \\
\rho v \\
E
\end{array}\right], F=\left[\begin{array}{c}
\rho u \\
\rho u^{2}+p \\
\rho u v \\
E u+p u
\end{array}\right], G=\left[\begin{array}{c}
\rho v \\
\rho u v \\
\rho v^{2}+p \\
E v+p v
\end{array}\right] .
$$




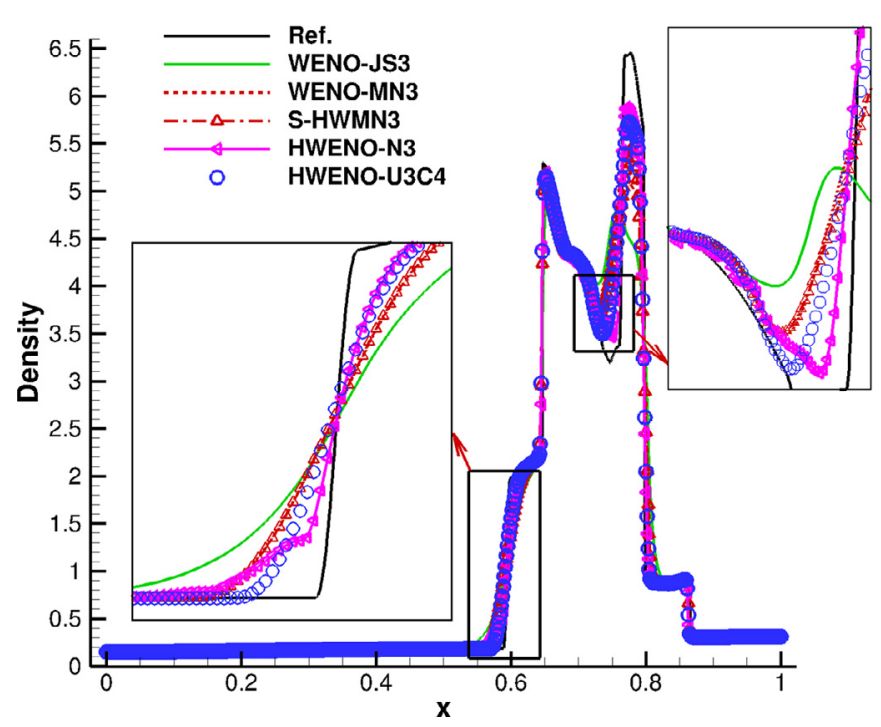

Fig. 22. Solutions of Case 7 computed by the WENO-JS3, WENO-MN3, S-HWMN3, HWENO-N3 and HWENO-U3C4 schemes with characteristic-wise reconstruction method.

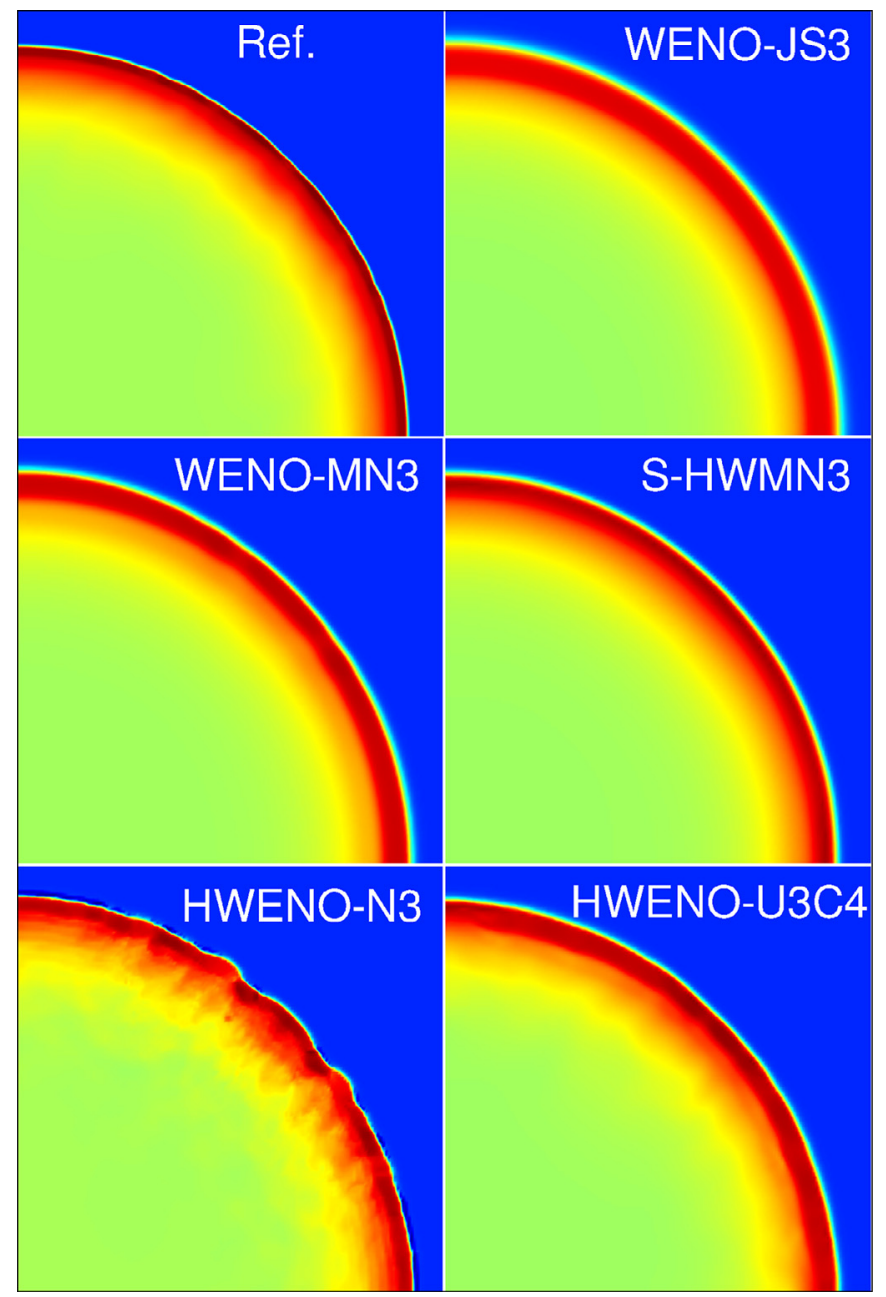

Fig. 23. Solutions of Case 8 computed by the WENO-JS3, WENO-MN3, S-HWMN3, HWENO-N3 and HWENO-U3C4 schemes with $150 \times 150$ at $t=1$ : pressure contours.

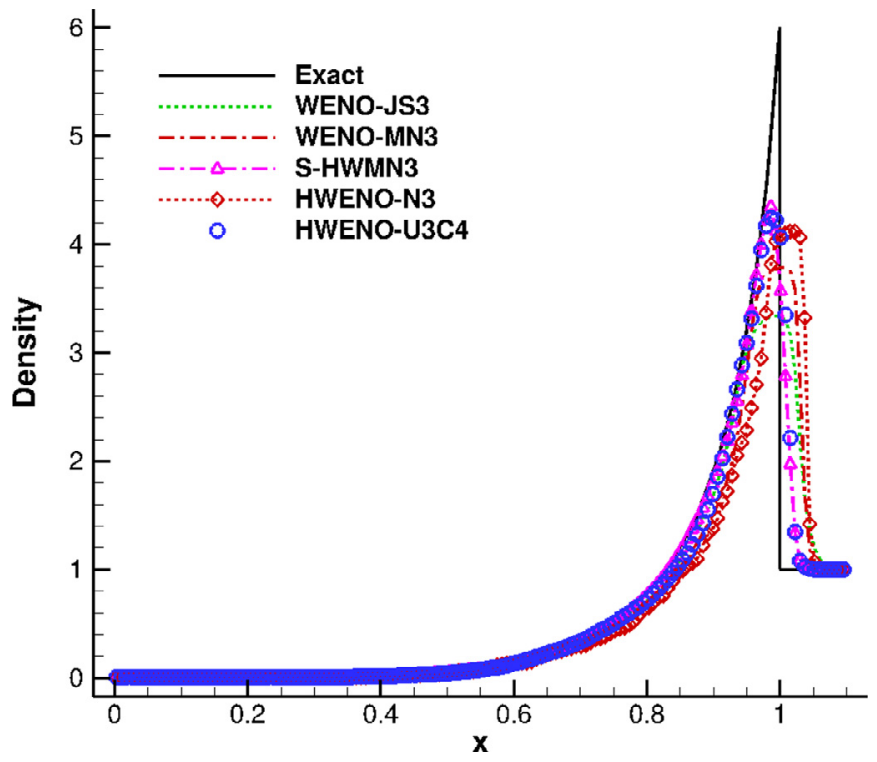

Fig. 24. Density distribution at $y=0$ of Case 8 computed by the WENO-JS3, WENOMN3, S-HWMN3, HWENO-N3 and HWENO-U3C4 schemes with $150 \times 150$ at $t=1$.

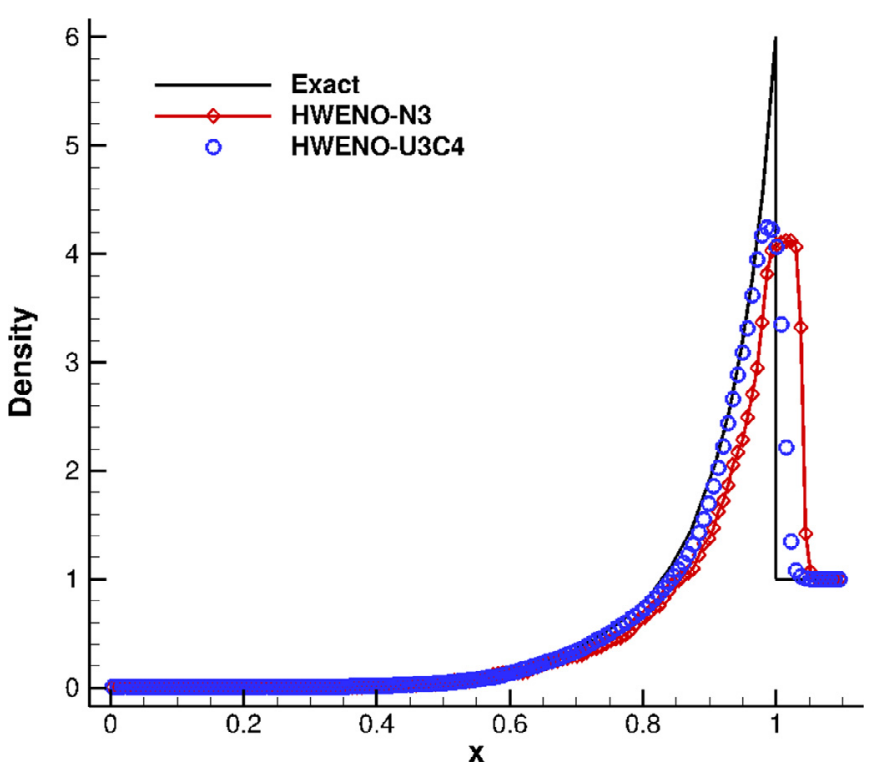

Fig. 25. Density distribution at $y=0$ of Case 8 computed by the HWENO-N3 and HWENO-U3C4 schemes with $150 \times 150$ at $t=1$.

where,

$E=\frac{p}{\gamma-1}+\frac{\rho}{2}\left(u^{2}+v^{2}\right)$

The time step is taken as follows,

$$
\begin{aligned}
\Delta t & =\sigma \frac{\Delta t_{x} \Delta t_{y}}{\Delta t_{x}+\Delta t_{y}}, \\
\Delta t_{x} & =\frac{\Delta x}{\max _{i, j}\left(\left|u_{i, j}\right|+c_{i, j}\right)}, \\
\Delta t_{y} & =\frac{\Delta y}{\max _{i, j}\left(\left|v_{i, j}\right|+c_{i, j}\right)} .
\end{aligned}
$$

where, $\sigma=0.5$ is CFL number. In this section, the global LF flux splitting method is used, and the Sedov blast wave problem [56], Riemann problem [8,57], double Mach reflection problem [58], Rayleigh-Taylor instability problem $[59,60]$ and the vortex propagation problem [61] are simulated to validate the shock captur- 


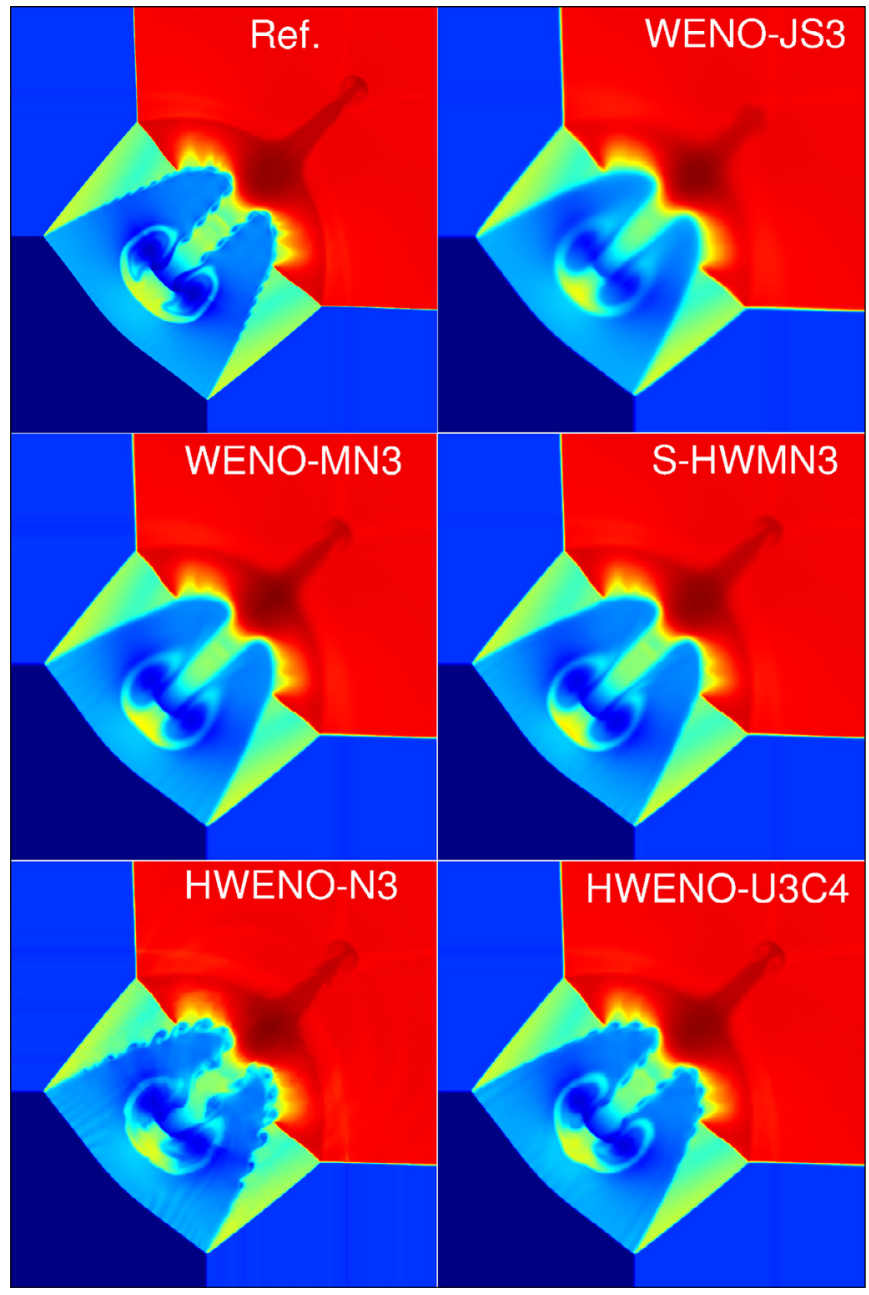

Fig. 26. Solutions of Case 9 computed by the WENO-JS3, WENO-MN3, S-HWMN3, HWENO-N3 and HWENO-U3C4 schemes with $400 \times 400$ at $t=0.8$ : density contours.

ing ability and the low dissipation property of the HWENO-U3C4 scheme.

\subsubsection{Case 8}

The Sedov blast wave problem [56] is a well known benchmark test to study a strong explosion problem. The initial conditions is given as follows,

$(\rho, u, v, E)= \begin{cases}\left(1,0,0,10^{-12}\right) & \text { if } x>\Delta x, y>\Delta y \\ \left(1,0,0, \frac{0.244816}{\Delta x \Delta y}\right) & \text { else. }\end{cases}$

The computational domain is $[0,1.1] \times[0,1.1]$. The numerical boundary treatment is reflective for the left and bottom edges, zero-order extrapolation for the right and top edges. The pressure contours and the density distribution along $x$-axis at $y=0$ at $t=1$ with $150 \times 150$ is given in Figs. 23-25, the reference solution in Fig. 23 is obtained by WENO-MN3 with $600 \times 600$. It can be seen that, the HWENO-U3C4 scheme resolved the strong shock pretty well. The HWENO-N3 scheme generated oscillations near the strong shock regions, and hence obtained wrong propagation speed near discontinuity.

\subsubsection{Case 9}

This is one of the two dimensional Riemann problems from $[8,57]$ with the initial conditions,

$(\rho, u, v, p)=$

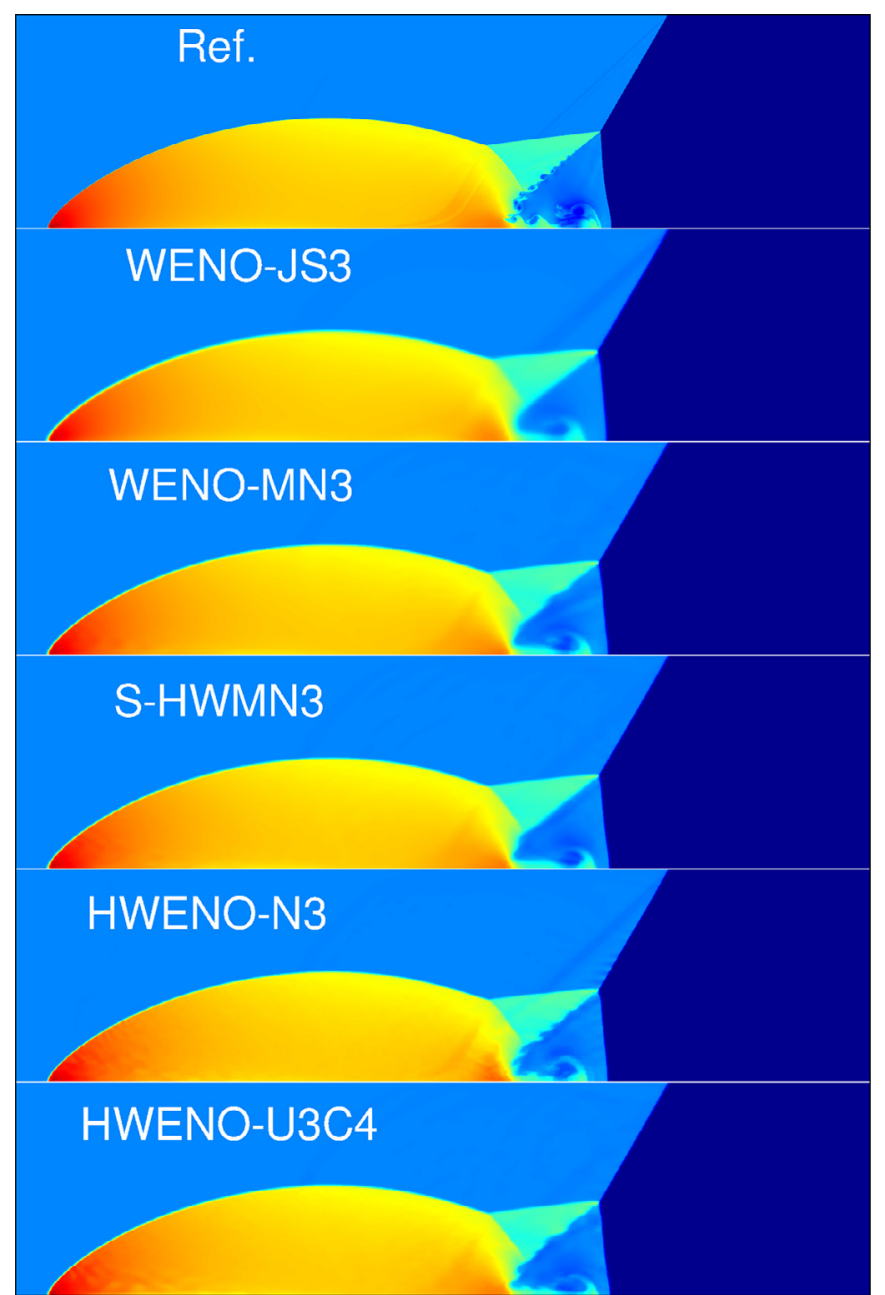

Fig. 27. Solutions of Case 10 computed by the WENO-JS3, WENO-MN3, S-HWMN3, HWENO-N3 and HWENO-U3C4 schemes with $800 \times 200$ at $t=0.2$ : density contours.

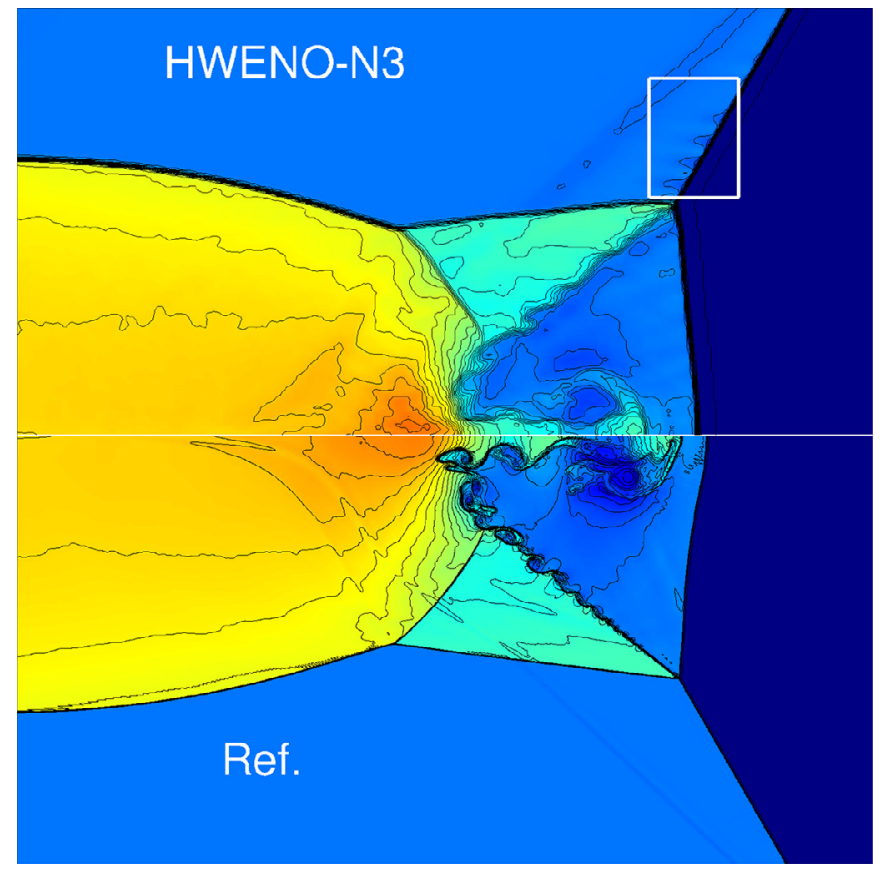

Fig. 28. Comparison of the results of HWENO-N3 and Ref. in Fig. 27, 50 density isolines from 1.4 to 23.8 . 


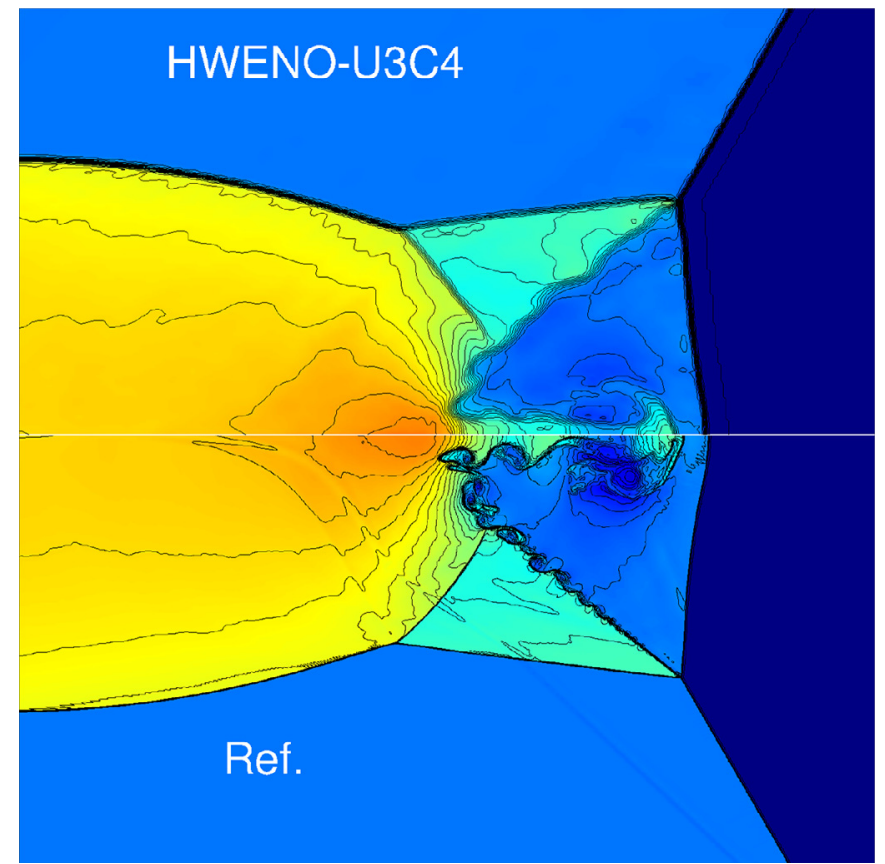

Fig. 29. Comparison of the results of HWENO-U3C4 and Ref. in Fig. 27, 50 density isolines from 1.4 to 23.8 .

$\begin{cases}(1.5,0,0,1.5) & 0.8 \leq x \leq 1,0.8 \leq y \leq 1 \\ (0.5323,1.206,0,0.3) & 0 \leq x<0.8,0.8 \leq y \leq 1, \\ (0.138,1.206,1.206,0.029) & 0 \leq x<0.8,0 \leq y<0.8 \\ (0.5323,0,1.206,0.3) & 0.8 \leq x \leq 1,0 \leq y<0.8\end{cases}$

The density contours at $t=0.8$ with $400 \times 400$ are shown in Fig. 26, the reference solution (Ref.) is calculated by the WENO-
MN3 scheme with $1000 \times 1000$. As we can see, the HWENOU3C4 scheme resolved the roll-up of the Kelvin-Helmholtz instability with finer structures and the HWENO-N3 scheme generates apparent asymmetric structures near discontinuities.

\subsubsection{Case 10}

This is a double Mach reflection problem [58] used to test the ability of shock capturing and the small scale structure resolution of high-order schemes. The computational domain is $[0,4] \times[0,3]$. At $t=0$, a right-moving Mach 10 shock with 60 degrees relative to the $x$-axis is positioned at $(1 / 6,0)$. Reflecting wall (i.e., only the normal velocity takes reflecting value, the density, pressure and the stream-wise velocity are extrapolated) starting from $x=1 / 6$ for bottom edge and zero-order extrapolation for right edge are implemented. The top edge is set to describe the exact motion of the Mach 10 shock. The density contours at $t=0.2$ with $800 \times 200$ is plotted in Fig. 27. Figs. 28 and 29 are the enlarged plot near Mach stem regions, the reference solution is computed by WENO-MN3 scheme with $3200 \times 800$. As shown in these figures, the result of HWENO-N3 shows obvious numerical noises [26] and oscillations near the shock regions, while the flow structures obtained by the HWENO-U3C4 scheme are much closer to the reference.

\subsubsection{Case 11}

The two-dimensional Rayleigh-Taylor instability problem $[59,60]$ is often used to test the low dissipation of a high-order scheme. It describes the interface instability between fluids with different densities when acceleration is directed from the heavy fluid to the light one. The gravitational effect is introduced by adding $\rho$ and $\rho v$ to the flux of the $y$-momentum and the energy equations, respectively. The initial distribution is,

$(\rho, u, v, p)= \begin{cases}(2,0,-0.025 \alpha \cos (8 \pi x), 2 y+1), & 0 \leq y<1 / 2, \\ (1,0,-0.025 \alpha \cos (8 \pi x), y+3 / 2), & 1 / 2 \leq y<1,\end{cases}$

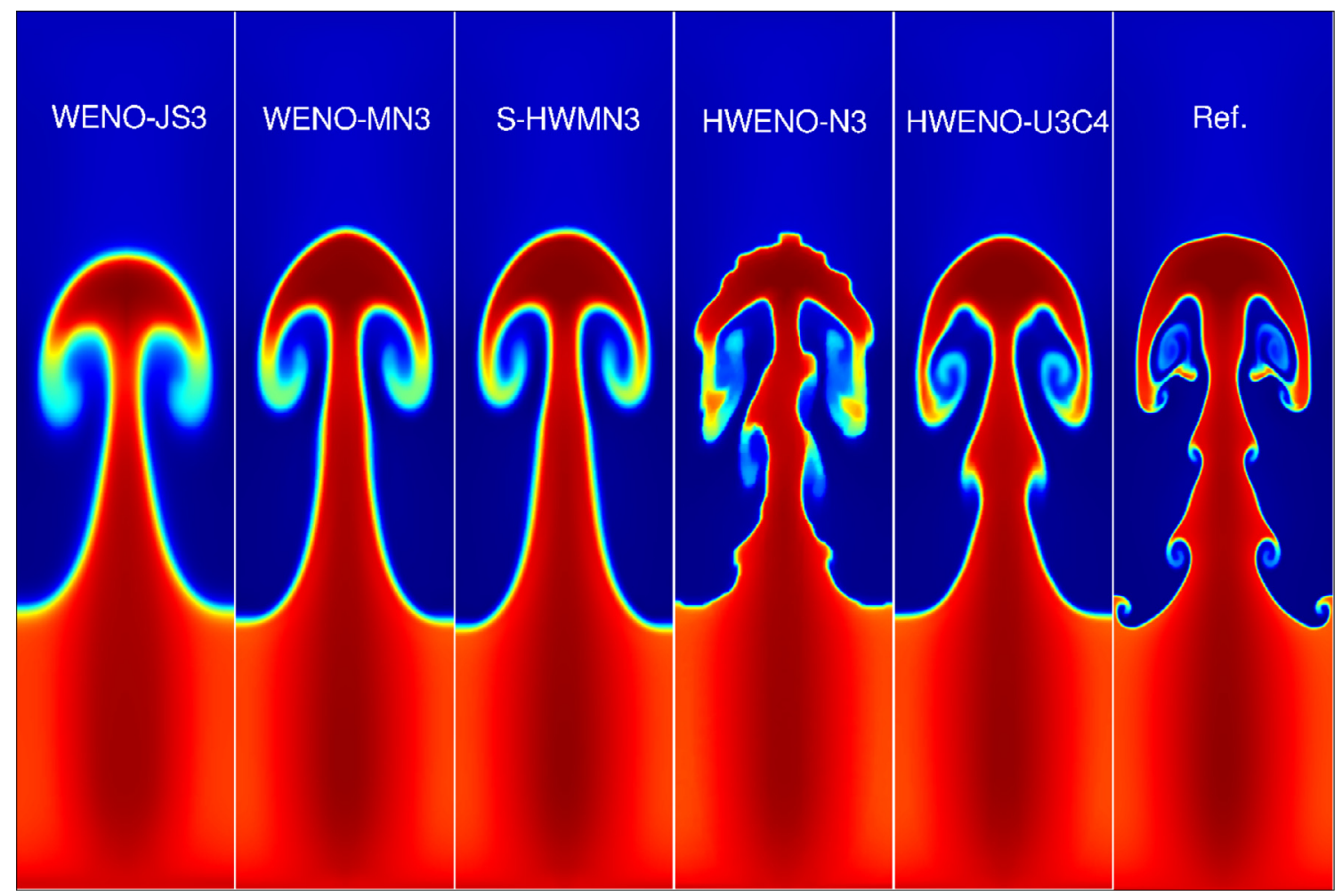

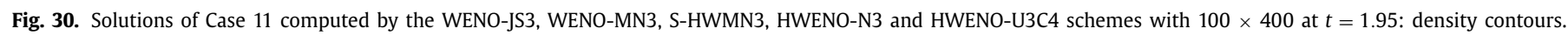




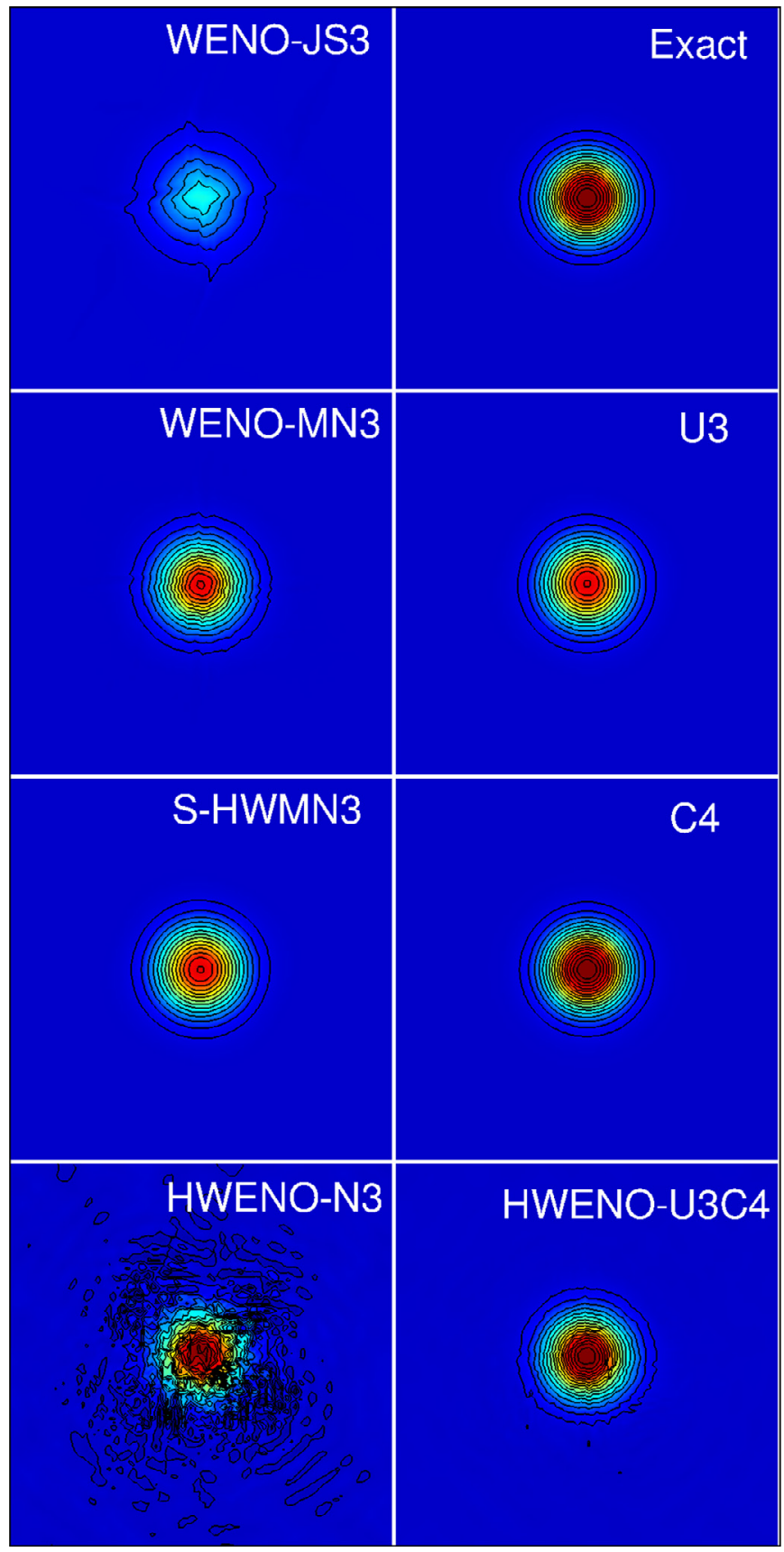

Fig. 31. Solutions of Case 12 computed by the U3, C4, WENO-JS3, WENO-MN3, SHWMN3, HWENO-N3 and HWENO-U3C4 schemes with $100 \times 100$ at $t=20$ : pressure contour lines from 0.991937 to 1.00102 .

and $\alpha=\sqrt{\gamma p / \rho}$ is the speed of sound with $\gamma=5 / 3$. The computational domain is $[0,0.25] \times[0,1]$. The left and right boundaries are reflective boundary conditions, and the top and bottom boundaries are set as $(\rho, u, v, p)=(1,0,0,2.5)$ and $(\rho, u, v, p)=(2,0,0,1)$, respectively. The solution at $t=1.95$ is solved with $100 \times 400$. The solution of WENO-MN3 with $400 \times 1600$ is given as a reference. The density contours are plotted in Fig. 30. As observed in previous cases, due to less dissipation, the HWENO-U3C4 scheme generates more complex unstable structures than the others. Similar to the Riemann problems, the HWENO-N3 scheme obtained apparent asymmetric structures.

The computational times of each scheme for this case are given in Table 3. The tests are carried out with a $2.9 \mathrm{GHz}$ Intel Core i7 in Fortran program. It reveals that the HWENO-U3C4 scheme needs

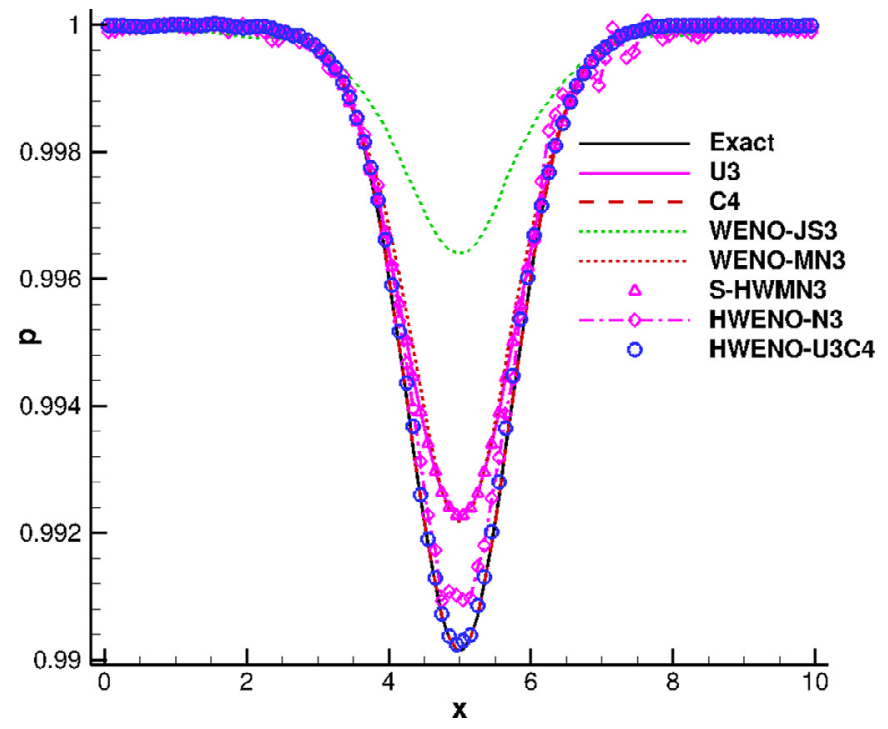

Fig. 32. Pressure distribution at $y=5$ of Case 12 computed by the U3, C4, WENO-JS3, WENO-MN3, S-HWMN3, HWENO-N3 and HWENO-U3C4 schemes with $100 \times 100$ at $t=20$.

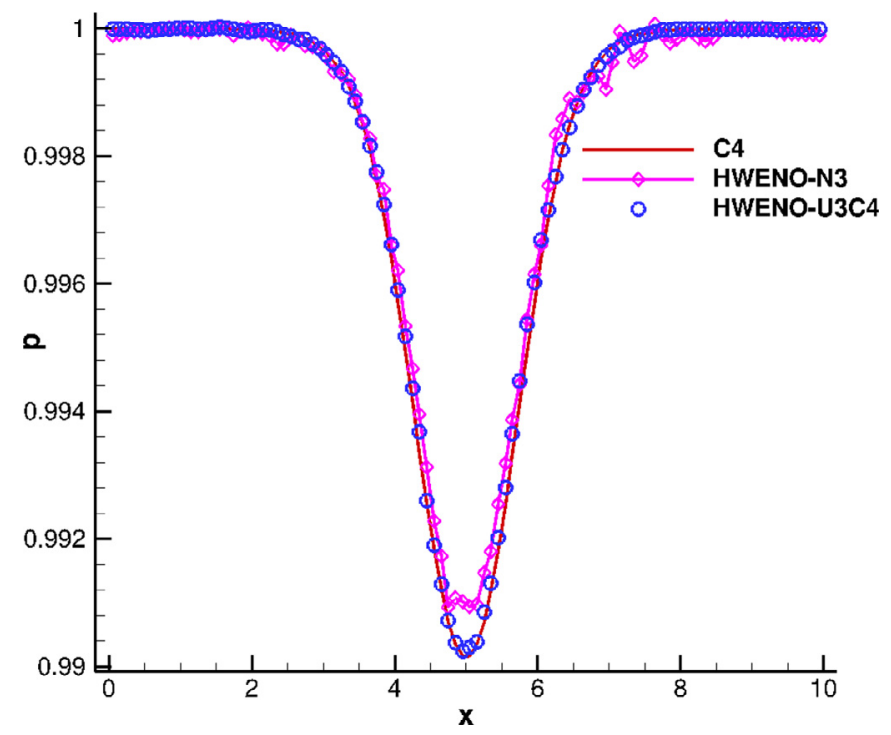

Fig. 33. Pressure distribution at $y=5$ of Case 12 computed by the C4, HWENO-N3 and HWENO-U3C4 schemes with $100 \times 100$ at $t=20$.

about 7\% more computational time than the S-HWMN3 scheme, while the HWENO-N3 scheme costs much more time than the others since the time-consuming weight function Eq. (12) is used (Ref. [39] also mentioned this issue).

\subsubsection{Case 12}

This is a two-dimensional periodic vortex propagation problem [61] used to assess the numerical dissipation of different schemes. The vortex is described as a perturbation to the velocity $(u, v)$, temperature $(T=p / \rho)$ and entropy $(S=p / \rho)$ of the mean flow $(\rho, u, v, p)=(1,0.5,0.5,1)$,

$$
\left\{\begin{array}{l}
\hat{u}=-\frac{\epsilon e^{\left(1-r^{2}\right) / 2}}{2 \pi} \bar{y} \\
\hat{v}=\frac{\epsilon e^{\left(1-r^{2}\right) / 2}}{2 \pi} \bar{x} \\
\hat{T}=-\frac{(\gamma-1) \epsilon^{2} e^{\left(1-r^{2}\right)}}{8 \gamma \pi^{2}} \\
\hat{S}=0
\end{array}\right.
$$


Table 3

Computational time of each scheme for Case 11 in seconds.

\begin{tabular}{llllll}
\hline & WENO-JS3 & WENO-MN3 & S-HWMN3 & HWENO-N3 & HWENO-U3C4 \\
\hline CPU-TIME & 472,794 & 512,761 & 495,039 & $1,317,351$ & 529,477 \\
efficiency & 1.00 & 1.08 & 1.05 & 2.79 & 1.12 \\
\hline
\end{tabular}

where, $(\bar{x}, \quad \bar{y})=(x-5, \quad y-5), \quad r^{2}=\bar{x}^{2}+\bar{y}^{2}, \quad$ and the vortex strength $\epsilon=0.5$. The computational domain is $[0,10] \times[0,10]$, and periodic boundary condition is used in both directions. It can be readily verified that the Euler equations with the above initial conditions admit an exact solution that moves with the speed $(0.5,0.5)$ in the diagonal direction. The results are carried out until $t=20$ with a grid of $100 \times 100$, to show the low dissipation of the HWENO-U3C4 scheme. Figs. 31-33 show the pressure contours and the pressure distribution along $x$-axis at $y=5$, respectively. Since S-HWMN3 almost reaches the third order accuracy, the waves can keep well. However, WENO-MN3 is only first order near critical points and has large dissipation, hence, with the time evolution, the waves are distorted. And it also can be seen that, the HWENOU3C4 scheme has the lowest dissipation and is very close to the central scheme. The HWENO-N3 scheme generates spurious waves and oscillations, even for the smooth flow field.

\section{Conclusion remarks}

This paper shows that the weighted hybrid scheme (HWENON3) of the two sub-schemes, e.g., the third-order WENO-N3 scheme and the fourth-order central scheme, may generate spurious waves near shock regions. So may the hybrid WENO scheme (HWENO-C4) combined with the WENO-MN3 and fourth-order central schemes by using discontinuity-detecting method directly. In order to overcome this problem, in this paper, a new two-step method is proposed for constructing a fourth-order hybrid central WENO scheme.

Numerical results show that, the new hybrid scheme (HWENOU3C4) can achieve fourth-order accuracy in $L_{1}$ errors in smooth regions including first critical point, keeps the property of essentially non-oscillatory, and also has low numerical dissipation.

It is worthy to note that, although only the hybrid scheme of a third-order WENO scheme and a fourth-order central scheme is studied in this paper, the two-step method can be easily implemented for higher-order hybrid central WENO schemes, too.

\section{Declaration of Competing Interest}

The authors declare that they have no known competing financial interests or personal relationships that could have appeared to influence the work reported in this paper.

\section{CRediT authorship contribution statement}

Shengping Liu: Writing - review \& editing, Writing - original draft, Conceptualization. Yiqing Shen: Supervision, Funding acquisition. Jun Peng: Software, Validation, Investigation. Jun Zhang: Project administration, Validation.

\section{Acknowledgments}

This research work was supported by the National Natural Science Foundation of China under grants NSFC Nos. 11872067 and 91852203, NKRDPC 2016YFA0401200 and SCP No. TZ2016002.

\section{Supplementary material}

Supplementary material associated with this article can be found, in the online version, at 10.1016/j.compfluid.2020.104590

\section{References}

[1] Shu CW. High order WENO and DG methods for time-dependent convectiondominated PDEs: a brief survey of several recent developments. J Comput Phys 2016;316:598-613.

[2] Liu X-D, Osher S, Chan T. Weighted essentially non-oscillatory schemes. J Comput Phys 1994;115:200-12.

[3] Jiang G-S, Shu CW. Efficient implementation of weighted ENO schemes. J Comput Phys 1996;126:202-28.

[4] Balsara DS, Shu CW. Monotonicity preserving weighted essentially non-oscillatory schemes with increasingly high order of accuracy. J Comput Phys 2000; $160: 405-52$

[5] Gerolymos G, Senechal D, Vallet I. Very-high-order WENO schemes. J Comput Phys 2009;228:8481-524.

[6] Henrick AK, Aslam TD, Powers JM. Mapped weighted essentially non-oscillatory schemes: achieving optimal order near critical points. J Comput Phys 2005;207:542-67.

[7] Borges R, Carmona M, Costa B, Don WS. An improved weighted essentially non-oscillatory scheme for hyperbolic conservation laws. J Comput Phys 2008;227:3191-211.

[8] Ha Y, Kim CH, Lee YJ, Yoon J. An improved weighted essentially non-oscillatory scheme with a new smoothness indicator. J Comput Phys 2013;232:68-86.

[9] Fan P, Shen Y-Q, Tian B-L, Yang C. A new smoothness indicator for improving the weighted essentially non-oscillatory scheme. J Comput Phys 2014;269:329-54.

[10] Kim CH, Ha Y, Yoon J. Modified non-linear weights for fifth-order weighted essentially non-oscillatory schemes. J Sci Comput 2016;67:299-323.

[11] Acker F, de R Borges RB, Costa B. An improved WENO-Z scheme. J Comput Phys 2016;313:726-53.

[12] Liu S, Shen Y, Zeng F, Yu M. A new weighting method for improving the WENO-Z scheme. Int J Numer Methods Fluids 2018;87:271-91.

[13] Marcos C, Bruno C, Sun DW. High order weighted essentially non-oscillatory WENO-Z schemes for hyperbolic conservation laws. J Comput Phys 2011;230:1766-92.

[14] Shen Y, Liu L, Yang Y. Multistep weighted essentially non-oscillatory scheme. Int J Numer Methods Fluids 2014;75:231-49.

[15] Ma Y, Yan Z, Zhu H. Improvement of multistep WENO scheme and its extension to higher orders of accuracy. Int J Numer Methods Fluids 2016;82:818-38.

[16] Zeng F, Shen Y, Liu S, Liu L. A high performance fifth-order multistep WENO scheme. Int J Numer Methods Fluids 2019;91:159-82.

[17] Hejranfar K, Saadat MH, Taheri S. High-order weighted essentially nonoscillatory finite-diference formulation of the lattice Boltzmann method in generalized curvilinear coordinates. Phys Rev E 2017:95:23314.

[18] Hejranfar K, Ezzatneshan E. A high-order compact finite-difference lattice Boltzmann method for simulation of steady and unsteady incompressible flows. Int J Numer Methods Fluids 2014;75:713-46.

[19] Shi X, Lin J, Yu Z. Discontinuous Galerkin spectral element lattice Boltzmann method on triangular element. Int J Numer Methods Fluids 2003;42:1249-61.

[20] Shen Y, Zha G. Improvement of the WENO scheme smoothness estimator. Int J Numer Methods Fluids 2010;64:653-75

[21] Xu Z, Shu CW. Anti-diffusive flux corrections for high order finite difference WENO schemes. J Comput Phys 2005;205:458-85.

[22] Huang C, Chen LL. A simple smoothness indicator for the WENO scheme with adaptive order. J Comput Phys 2018;352:498-515

[23] Johnsen E, Colonius T. Implementation of WENO schemes in compressible multicomponent flow problems. J Comput Phys 2006;219:715-32.

[24] He Z, Zhang Y, Li X, Li L, Tian B. Preventing numerical oscillations in the flux-split based finite differece method for compressible flows with discontinuities. J Comput Phys 2015;300:269-87.

[25] Zhao S, Lardjane N, Fedioun I. Comparison of improved finite-difference WENO schemes for the implicit large eddy simulation of turbulent non-reacting and racting high-speed shear flows. Comput Fluids 2014;95:74-87.

[26] Peng J, Zhai C, Ni G, Yong H, Shen Y. An adaptive characteristic-wise reconstruction WENO-z scheme for gas dynamic euler equations. Comput Fluids 2019;179:34-51.

[27] Kim D, Kwon JH. A high-order accurate hybrid scheme using a central flux scheme and a WENO scheme for compressible flowfield analysis. J Comput Phys 2005;210:554-83.

[28] Costa B, Don WS. High order hybrid central - WENO finite difference scheme for conservation laws. J Comput Appl Math 2007;204:209-18.

[29] Hu X, Wang Q, Adams N. An adaptive central-upwind weighted essentially non-oscillatory scheme. J Comput Phys 2010;229:8952-65.

[30] Huang C, Chen LL. A new adaptively central-upweind sixth-order WENO scheme. J Comput Phys 2018;357:1-15.

[31] Yamaleev NK, Carpenter MH. A systematic methodology for constructing high-order energy stable WENO schemes. J Comput Phys 2009;228:4248-72. 
[32] Hu F. The 6th-order weighted ENO schemes for hyperbolic conservation laws. Comput Fluids 2018;174:34-45.

[33] Martin M, Taylor E, Wu M, Weirs V. A bandwidth-optimized WENO scheme for the effective direct numerical simulation of compressible turbulence. J Comput Phys 2006;220:270-89.

[34] Adams N, Shariff K. A high-resolution hybrid compact-ENO scheme for shockturbulence interaction problems. J Comput Phys 1996;127:27-51.

[35] Ren Y, Liu M, Zhang H. A characteristic-wise hybrid compact-WENO scheme for solving hyperbolic conservation laws. J Comput Phys 2003;192:365-86.

[36] Liu S, Shen Y, Chen B, Zeng F. Novel local smoothness indicators for improving the third-order WENO scheme. Int J Numer Methods Fluids 2018;87:51-69.

[37] Liu S, Shen Y. Discontinuity-detecting method for a four-point stencil and its application to develop a third-order hybrid-WENO scheme. J Sci Comput 2019;81:1732-66.

[38] Yamaleev NK, Carpenter MH. Third-order energy stable WENO scheme. J Comput Phys 2009;228:3025-47.

[39] Wu X, Zhao Y. A high-resolution hybrid scheme for hyperbolic conservation laws. Int J Numer Methods Fluids 2015;78:162-87.

[40] Wu X, Lian J, Zhao Y. A new smoothness indicator for third-order WENO scheme. Int J Numer Methods Fluids 2016;81:451-9.

[41] Gande N, Rathod Y, Samala R. Third-order WENO scheme with a new smoothness indicator. Int J Numer Methods Fluids 2017;85:90-112.

[42] Gande N, Rathod Y, Samala R. Improved third-order weighted essentially non-oscillatory scheme. Int J Numer Methods Fluids 2018;87:329-42.

[43] Bhise AA, Naga Raju G, Samala R, Devakar M. An efficient hybrid WENO scheme with a problem independent discontinuity locator. Int J Numer Methods Fluids 2019;91:1-28.

[44] Xu W, Wu W. An improved third-order WENO-z scheme. J Sci Comput 2018;75:1808-41.

[45] Xu W, Wu W. An improved third-order weighted essentially non-oscillatory scheme achieving optimal order near critical points. Comput Fluids 2018;162:113-25

[46] Li G, Qiu J. Hybrid weighted essentially non-oscillatory schemes with different indicators. J Comput Phys 2010;229:8105-29.
[47] Li G, Qiu J. Hybrid WENO schemes with different indicators on curvilinear grids. Adv Comput Math 2014;40:747-72.

[48] Peng J, Shen Y. A novel weighting weitch function for uniformly high-order hybrid shock-capturing schemes. Int J Numer Methods Fluids 2017;83:681-703.

[49] Shen Y, Yang G. Hybrid finite compact-WENO schemes for shock calculation. Int J Numer Methods Fluids 2007;53:531-60.

[50] Wang R, Spiteri RJ. Linear instability of the fifth-order WENO method. SIAM J Numer Anal 2007; 45:1871-901.

[51] Motamed M, Macdonald CB, Ruuth SJ. On the linear stability of the fifth-order WENO discretization. J Sci Comput 2011;47:127-49.

[52] Hermes V, Klioutchnikov I, Olivier H. Linear stability of WENO schemes coupled with explicit Runge-Kutta schemes. Int J Numer Methods Fluids 2012;69:1065-95.

[53] Shu C-W, Osher S. Efficient implementation of essentially non-oscillatory shock-capturing schemes. J Comput Phys 1988;77:439-71.

[54] Najafi-Yazdi A, Mongeau L. A low-dispersion and low-dissipation implicit Runge-Kutta scheme. J Comput Phys 2013;233:315-23.

[55] Shu C. Essentially non-oscillatory and weighted essentially non-oscillatory schemes for hyperbolic conservation law. ICASE Report; 1998.

[56] Zhang X, Shu CW. Positivity-preserving high order finite difference WENO schemes for compressible Euler equations. J Comput Phys 2012;231:2245-58.

[57] Balsara DS. Multidimensional HLLE Riemann solver: application to euler and magnetohydrodynamic flows. J Fluid Mech 2010;229:1970-93.

[58] Woodward P, Colella P. The numerical simulation of two-dimensional fluid flow with strong shocks. J Comput Phys 1984;54:115-73.

[59] Shi J, Zhang YT, Shu CW. Resolution of high order WENO schemes for complicated flow structures. J Comput Phys 2003;186:690-6.

[60] Yong YN, Tufo H, dubey A, Rosner R. On the miscible rayleigh-taylor instability: two and three dimensions. J Fluid Mech 2001;447:337-408.

[61] Sun Y, Wang Z. Evaluation of discontinuous Galerkin and spectral volume methods for scalar and system conservation laws on unstructured grids. Int J Numer Methods Fluids 2004;45:819-38. 